\title{
One - Memory in Repeated Games*
}

\author{
Mehmet Barlo \\ Sabancı University
}

\author{
Guilherme Carmona \\ Universidade Nova de Lisboa
}

Hamid Sabourian

University of Cambridge

November 17, 2006

\begin{abstract}
We study the extent to which equilibrium payoffs of discounted repeated games can be obtained by 1 - memory strategies. First, we present robust examples of games in which there is a subgame perfect equilibrium payoff profile that cannot be obtained by any 1 memory subgame perfect equilibrium. Then, a complete characterization of 1 - memory simple strategies is provided, and it is employed to establish the following in games with more than two players each having connected action spaces:

1. all subgame perfect equilibrium payoffs can be approximately supported by an $\varepsilon$ - subgame perfect equilibrium strategy of 1 - memory,

2. all strictly enforceable subgame perfect equilibrium payoffs can be approximately supported by a 1 - memory subgame equilibrium, and

3. the subgame perfect Folk Theorem holds for 1 - memory strategies.

While no further restrictions are needed for the third result to hold in 2 - player games, an additional restriction is needed for the first two: players must have common punishments.
\end{abstract}

Journal of Economic Literature Classification Numbers: C72; C73; C79

Keywords: Repeated Games; Memory; Bounded Rationality; Folk Theorem

${ }^{*}$ This is a revised version of chapter 2 in Barlo (2003), and of Sabourian (1989). We thank Beth Allen, Pedro Amaral, Kemal Badur, Kevin Hasker, Ehud Kalai, Narayana Kocherlakota, Andy McLennan, Han Özsöylev, Aldo Rusticini and Jan Werner and participants at the Applied Microeconomics Workshop and Mathematical Economics Workshop at the University of Minnesota, the Games 2002 conference, the Mid-West Mathematical Economics 2002 meeting, the SAET 2003 conference, and the ASSET 2003 and 2006 conferences for helpful comments and suggestions. Any remaining errors are ours. 


\section{Introduction}

The Folk Theorem of repeated games states that any individually rational payoffs can be sustained as an equilibrium if the players are sufficiently patient (see Fudenberg and Maskin (1986) and Aumann and Shapley (1994)). Such a multiplicity of equilibria arises because in repeated games at any stage each player can condition his behavior on the past behavior of all the players. Such long memories are clearly unreasonable.

Even when players are impatient, equilibrium strategies often require them to remember distant pasts. In fact, Abreu (1988) characterized the subgame perfect equilibrium outcome paths of discounted repeated games by using simple strategies which satisfy certain incentive conditions. Specifically, a simple strategy profile induces $n+1$ outcome paths (states): the given prescribed play, and a punishment path for each of the $n$ players. At any stage, unless there has been a single player deviation, simple strategies make the play continue along the given outcome path. In the case of a single player deviation, all the other players will punish the deviator with a player specific outcome path. Thus, in particular, the behavior at a given state of the game may depend unboundedly on the past. Therefore, because of the extensive memory dependence such simple equilibria are also often regarded as unappealing when compared with those in which the current behavior either does not depend on the past or depends at most on the behavior of the last few periods.

In this paper, we restrict the set of strategies to those that depend only on what has happened in the previous period. We shall refer to such behavior by 1 - memory strategies (in the literature they are also known by 1-period recall strategies). We then ask whether or not we can obtain the subgame perfect equilibrium (SPE) payoffs of complete information repeated games with 1 memory strategies. We address this issue for the case in which the set of actions available at any stage of the game is sufficiently "rich" (the stage game has a large number of actions). ${ }^{1}$

In Section 3, we first start with some repeated game examples with discounting showing that the answer to the above question is negative. These examples have SPE payoffs that cannot be

\footnotetext{
${ }^{1}$ Sabourian (1998) characterizes the set subgame perfect equilibria with bounded memory for the case of repeated games with no discounting and finite number of pure actions. Other works on repeated games with limited memory include Kalai and Stanford (1988), Lehrer (1988), Aumann and Sorin (1989), Lehrer (1994), Neyman and Okada (1999), Bhaskar and Vega-Redondo (2002) and Barlo and Carmona (2006).
} 
obtained with 1 - memory strategies. Moreover, these negative results are robust to perturbations of stage game payoffs and discount factor. We also show that the equilibrium in the 2 - player example cannot even be approximately supported as an $\varepsilon$ - contemporaneous perfect equilibrium $(\varepsilon-\mathrm{CPE})$ with $1-$ memory. $^{2}$

Despite these strong negative examples, we demonstrate in the rest of the paper that the answer to the above question is affirmative with some appropriate qualifications. Our main results demonstrate, at least for repeated games with more than 2 players, that strategies with 1-period memory are approximately enough to obtain all SPE payoffs of discounted repeated games if the action space at each stage is sufficiently rich. More specifically, if the action spaces are connected then our approximation results hold, for games with more than two players, in the following three senses. First, any SPE payoff profile can be supported with a $1-$ memory $\varepsilon-\mathrm{CPE}$, for all $\varepsilon>0$. Second, if an SPE payoff profile is sustained by simple strategies with the property that the incentive conditions identified by Abreu (1988) hold strictly, then it can be approximated by a 1 memory SPE. Third, for generic games, in the limit as the discount factor converges to one, any strictly individually rational payoff can be approximated by a 1 - memory SPE. We also show that a similar result holds without the genericity assumption for the no discounting case. Furthermore, if 1 - memory strategies can be conditioned on time, the limiting result for the case of the discount factor converging to one is exact and not only an approximation.

The last sets of results with patient players also hold for 2 - player games. However, as the 2 - player example in Section 3 demonstrates, with arbitrary discount factors, the first two sets of approximation results obtained for games with more than two players (the $\varepsilon-\mathrm{CPE}$ approximation and the approximation of simple strategy equilibria that satisfy the Abreu type incentive conditions strictly) do not necessarily extend to 2 - player games without further assumptions on the structure of the equilibria. In Section 5, we demonstrate that these two approximation results also hold for 2 - player games if the equilibrium considered is such that the punishment path induced when one player deviates is the same as that induced when the other player deviates.

These results, then, suggest, at least for games with more than two player, that the restriction to

\footnotetext{
${ }^{2}$ The notion of contemporaneous perfect epsilon equilibrium, introduced and analyzed by Mailath, Postlewaite, and Samuelson (2005), demands that at every history no single handed deviation pays strictly more than $\varepsilon$ than the equilibrium continuation payoff.
} 
1 - memory strategies will not place severe limitations on equilibrium payoffs: It is approximately enough for players to remember what has happened in the previous period in order to obtain any SPE payoff. Furthermore, our results also demonstrate that the Folk Theorem does not depend on the ability of players to remember more than the previous period. Thus, as long as players remember the last period imposing bounds on memory does not reduce the abundance of equilibrium payoffs.

Clearly, not all strategies are 1 - memory strategies. For any equilibrium payoff profile, our approach involves employing simple strategies which first can be implemented by remembering only what has happened in the previous period, and second induce (approximately) the same equilibrium profile. This requires each agent to identify the state of the play with 1 - memory. Such decoding of the state of play by observing the outcome in the previous period is clearly possible with some strategies. For example, consider the grim-trigger strategy profile in the infinitely repeated version of the Prisoner's Dilemma. Since in every period players need to know whether or not someone defected in the first period, such a strategy profile has infinite memory. However, note that the outcome path it induces can clearly be supported by the following 1 - memory strategy profile: players start by cooperating; in the following stages of the game, each player cooperates if and only if they both have cooperated in the previous stage. A more complicated example would be a path that involves players playing the same action profile $a$ for a finite number of periods $T$ followed by playing another profile $b$ forever. Again, this path requires at least $T$ - memory in order to know when to switch to $b$. However, if there exists $T$ distinct action profiles $a^{1}, \ldots, a^{T}$ each generating payoffs close to those by $a$, then the path that plays the sequence $a^{1}, \ldots, a^{T}$ followed by playing $b$ forever approximates the original path in terms of payoffs and is implementable with 1 - memory.

This second example indicates that a path may be approximately implementable with 1 - memory if the set of action profile is sufficiently rich. However, to implement an (equilibrium) strategy profile with 1 - memory, we need a great deal more than just being able to implement a specific path. For example, in the case of a simple strategy profile, not only the equilibrium paths and all the $n$ punishment paths (where $n$ denotes the number of the players) need to be implementable with 1 - memory, but also it should be the case that (i) the action profiles used in the punishment phase for any player occurs neither on the equilibrium path nor be used in the punishment phase for other players, and (ii) any single deviation can be detected by observing the previous period. ${ }^{3}$

\footnotetext{
${ }^{3}$ For instance, it must be the case that a player being punished cannot, by deviating from the action that the
} 
Otherwise, it may not be possible for players to know the state of play with 1 - memory.

More formally, to rule out such ambiguities, we introduce a critical property, the notion of confusion proofness of simple strategies. This notion turns out to be both necessary and sufficient for players to find out in which phase of the $n+1$ paths the play is in by observing only what has happened in the previous period. In particular, our Proposition 1 establishes that a simple strategy is 1 - memory if and only if it is confusion-proof.

We then establish our set of approximation results by showing that for any SPE payoff profile there exists an $(\varepsilon-)$ equilibrium confusion proof simple strategy profile that approximate the original equilibrium in the three senses mentioned above. In particular, in the discounting case, payoffs of non-confusion proof simple strategies are approximated by making use of the notion of $\varepsilon$ - strict enforceability of a simple strategy, for any $\varepsilon \geq 0$. This notion requires that at any date and state, every player loses less than $\varepsilon \geq 0$ by conforming with the simple strategy. Then, with the use of connected action spaces we employ this slack to construct a confusion proof simple strategy profile with a payoff arbitrarily close to the original one. Indeed, this construction is the key ingredient for our discounting Folk Theorem with 1 - memory. But, because correlated strategies are not allowed, the proof of our 1 - memory Folk Theorem becomes considerably more elaborate.

The above approach clearly cannot be applied when agents have a small (finite) number of actions at each stage of the game (in this case 1 - memory would not be enough to obtain our results — see Sabourian (1998)). On the other hand, rich (connected) action spaces endow the agents with the capacity to "code" information about who-deviated-when into their play; thereby, allow us to establish our results.

Notice, our richness of action space assumption is consistent with most standard games with infinite action spaces because it is often assumed that the action space is a convex (and hence connected) subset of some finite dimensional Euclidian space. Since the set of mixed strategies are also convex, it also follows that our richness assumption is also satisfied in any repeated game (with finite or infinite pure action space) in which at each stage the players are allowed to choose mixed strategies and past mixed actions are observable as in Aumann (1964). ${ }^{4}$

It is very important to point out that replacing memory with a complex set of actions is not punishments prescribe, give rise to an action profile on the equilibrium outcome.

${ }^{4}$ We use the term "mixed action" to denote individual randomization over the actions in the stage game. 
sufficient to obtain all equilibria even with a rich action space. The two repeated games examples, one with two and another with three players, in Section 3 demonstrate this, in addition to showing that the main difficulties and subtleties that can arise in implementing equilibria with 1 - memory. In both examples the action space for each player at any stage consists of the set of mixed strategies over two pure strategies and is therefore convex. Nevertheless, both have SPE payoffs that cannot be obtained by 1 - memory. These examples are generic since they remain valid for any small perturbations of the payoffs and/or the discount factor. Furthermore, the 2 - player equilibrium example cannot even be approximated by a $1-$ memory $\varepsilon-\mathrm{CPE}$. As a result there is a type of discontinuity associated with the 3 - player counter-example: even though it cannot be sustained with 1 - memory SPE strategies, it can be supported by a $1-$ memory $\varepsilon-\mathrm{CPE}$ for all $\varepsilon>0$.

The explanation for not being able to implement the equilibrium in the 3 - player example with 1 - memory and for the associated discontinuity is that there is no slack in the incentive conditions for the particular equilibrium payoff we consider; as a result there is no room to code information about the past into agents' behavior without violating the incentive conditions. The explanation for the 2 - player counter-example is that when behavior depend only on the outcome in the preceding periods, with two players and 1 - memory there are additional confusing instances that might arise that does not occur when the number of players exceed two: when $n>2$, it is considerably easier to identify single player deviations than it is in the 2 - player case. For instance, consider the following simple strategy in a 2 - player game: the equilibrium path consists of repetitions of an action profile $\left(a_{1}, a_{2}\right)$ and the punishment path for player 1 (resp., player 2) consists of repetitions of $\left(b_{1}, b_{2}\right)$ (resp., $\left.\left(c_{1}, c_{2}\right)\right)$. When players observe $\left(b_{1}, a_{2}\right)$ in the last period, they cannot conclude whether or not it was player 1 who deviated from the equilibrium path, or if it was player 2 who deviated from the punishment path of player 1 . This is clearly a problem unless both players have a common punishment path, in which case they do not need know who has deviated. In contrast, this confusing instance cannot arise with three or more players because, for any two players $i$ and $j$, it is always possible to use the last period actions of players other than $i$ and $j$ to find out if $i$ or $j$ has deviated in the previous period. ${ }^{5}$

\footnotetext{
${ }^{5}$ Nevertheless, confusing instances can still occur in 3 - player games. If a player being punished has an opportunity to mislead others to believe that in the previous period someone else was being punished (or that they were playing along the equilibrium path), then such a strategy is not immune to confusion, and therefore, is not 1 - memory.
} 
Several other papers study the effects of restricting the strategies players can use in repeated games. The most restrictive assumption is to consider 0 - memory strategies. In those, players play the same action profile in every period, independently of the past - this corresponds to the notion of stationary/Markov strategies. Clearly, the stationary subgame perfect equilibria are precisely those that consist of playing a Nash equilibrium of the stage game at any subgame. Consequently, it is quite surprising that the next step of dependence on the past, 1 - memory, is approximately enough to characterize all equilibrium payoffs.

Another important class of repeated strategies are those represented by finite automata. Similar results to the ones obtained here appeared in Kalai and Stanford (1988), as they have shown that all subgame equilibrium payoffs can be approximately supported by finite automata as an approximate equilibrium for sufficiently large automata. They do not assume that the action space is large because they allow any finite size automata. Our results are different because we only consider strategies with one period recall.

Memory in terms of recall used in this paper captures one aspect of complexity of a strategy. There are clearly other aspects of complexity of a strategy. We do not address these in this paper. In particular, we obtain our approximation results with 1 - memory/recall by using (cycle) paths that involve different action profiles at each date. Such paths may be complex if we use an alternative definition of complexity to the notion of memory (recall) we use in this paper. The objective here is not to tackle this general issue of complexity but simply to characterize the implications of recall restriction, and in particular, to explain how, with some qualifications, in repeated games with rich action spaces players do not need to use much memory: remembering yesterday is almost enough to support all SPE payoffs.

In Section 2, we provide the notation and the definitions. Section 3 presents two examples. Section 4 establishes when an outcome path can be obtained with the use of 1 - memory strategies. The discounting case is analyzed in Sections 5. In Section 6 we discuss our 1 - memory Folk Theorems. Finally, in Section 7, we consider time-dependent strategies. All the proofs are in the Appendix. 


\section{Notation and Definitions}

\section{The stage game:}

A normal form game $G$ is defined by $G=\left(N,\left(S_{i}\right)_{i \in N},\left(u_{i}\right)_{i \in N}\right)$, where $N$ is a finite set of players, $S_{i}$ is the set of player $i$ 's actions and $u_{i}: \prod_{i \in N} S_{i} \rightarrow \mathbb{R}$ is player $i$ 's payoff function.

We assume that $S_{i}$ is a connected and compact metric space and that $u_{i}$ is continuous for all $i \in N$. Note that if $S_{i}$ is convex, then $S_{i}$ is connected. Therefore, the mixed extension of any finite normal form game satisfies the above assumptions. ${ }^{6}$

Let $S=\prod_{i \in N} S_{i}$ and $S_{-i}=\prod_{j \neq i} S_{i}$. Also, for any $i \in N$ denote respectively the minmax payoff and a minmax profile for player $i$ by

$$
\begin{aligned}
& v_{i}=\min _{s_{-i} \in S_{-i}} \max _{s_{i} \in S_{i}} u_{i}\left(s_{i}, s_{-i}\right), \\
& m^{i} \in \arg \min _{s_{-i} \in S_{-i}} \max _{s_{i} \in S_{i}} u_{i}\left(s_{i}, s_{-i}\right) .
\end{aligned}
$$

If $G$ is a 2 - player game, a mutual minmax profile is $\bar{m}=\left(m_{1}^{2}, m_{2}^{1}\right)$.

\section{The repeated game:}

The supergame $G^{\infty}$ of $G$ consists of an infinite sequence of repetitions of $G$. We denote the action of any player $i$ in $G^{\infty}$ at any date $t=1,2,3, \ldots$ by $s_{i}^{t} \in S_{i}$. Also, let $s^{t}=\left(s_{1}^{t}, . ., s_{n}^{t}\right)$ be the profile of choices at $t$.

For $t \geq 1$, a $t$ - stage history is a sequence $h_{t}=\left(s^{1}, \ldots, s^{t}\right)$. The set of all $t$ - stage histories is denoted by $H_{t}=S^{t}$ (the $t$ - fold Cartesian product of $S$ ). We use $H_{0}$ to represent the initial $(0-$ stage) history. The set of all histories is defined by $H=\bigcup_{t=0}^{\infty} H_{t}$.

For all $i \in N$, player $i$ 's strategy is a function $f_{i}: H \rightarrow S_{i}{ }^{7}$ The set of player $i$ 's strategies is denoted by $F_{i}$, and $F=\prod_{i \in N} F_{i}$ is the joint strategy space with a typical element $f \in F$.

Given a strategy $f_{i} \in F_{i}$ and a history $h \in H$, denote the strategy induced at $h$ by $f_{i} \mid h$; thus $\left(f_{i} \mid h\right)(\bar{h})=f_{i}(h, \bar{h})$, for every $\bar{h} \in H$. Also, let $f \mid h=\left(f_{1}\left|h, \ldots, f_{n}\right| h\right)$ for every $f \in F$ and $h \in H$.

Any strategy $f \in F$ induces an outcome at any date as follows:

$$
\pi^{1}(f)=f\left(H_{0}\right) \text { and } \pi^{t}(f)=f\left(\pi^{1}(f), \ldots, \pi^{t-1}(f)\right) \text { for any } t>1 .
$$

\footnotetext{
${ }^{6}$ More generally, the mixed extension of any normal form game with compact metric strategy spaces and continuous payoff functions also satisfies the above assumptions.

${ }^{7}$ Notice that when $G$ refers to the mixed extension of a normal form game, then the strategy in the repeated game at any period may depend on past randomization choices which in such cases must be publicly observable.
} 
Denote the set of outcome paths by $\Pi=S \times S \times \cdots$ and define the outcome path induced by any strategy profile $f \in F$ by $\pi(f)=\left\{\pi^{1}(f), \pi^{2}(f), \ldots\right\} \in \Pi$.

We consider the following memory restriction on the set of strategies in this paper. For any history $h \in H$, the 1 - period tail of $h$ is $T(h)=s^{t}$ if $h=\left(s^{1}, \ldots, s^{t}\right)$ for some $t \geq 1$.

Definition 1 A strategy $f_{i} \in F_{i}$ has one period memory (henceforth called 1 - memory) if $f_{i}(h)=$ $f_{i}(\bar{h})$ for any two histories $h, \bar{h} \in H$ such that $T(h)=T(\bar{h})$.

Notice that in the above definition the choice of action at any date $t$ depends only on the last stage of the supergame and not $t$; thus, 1 - memory strategies are independent of the calendar time. We let $F_{i}^{1}$ be the set of all player $i$ 's strategies with 1 - memory, and $F^{1}=\prod_{i \in N} F_{i}^{1}$.

For all $i \in N$, let $U_{i}: F \rightarrow \mathbb{R}$ be player $i$ 's payoff function in the supergame of $G$.

A strategy vector $f \in F$ is a Nash equilibrium of $G^{\infty}$ if $U_{i}(f) \geq U_{i}\left(\hat{f}_{i}, f_{-i}\right)$ for all $i \in N$ and all $\hat{f}_{i} \in F_{i}$. A strategy vector $f \in F$ is a $S P E$ of $G^{\infty}$ if $f \mid h$ is a Nash equilibrium for all $h \in H$.

We also define a 1 - memory SPE as a SPE with the additional property that it has 1 - memory. ${ }^{8}$

\section{Two Examples}

In this section we present two examples of SPE payoffs that cannot be supported by any 1 - memory SPE strategy profiles. The first example uses a 2 - player game while the second uses one with 3 players.

\subsection{Two Players}

Consider the following normal form game:

\begin{tabular}{|c|c|c|}
\hline $1 \backslash 2$ & $a$ & $b$ \\
\hline$a$ & 4,4 & 2,5 \\
\hline$b$ & 5,2 & 0,0 \\
\hline
\end{tabular}

\footnotetext{
${ }^{8}$ Notice that with this definition the equilibrium strategy of each player has 1 - memory but is best amongst all strategies, including those with memory longer than one. Alternatively, we could have just required optimality amongst the set of 1 - memory strategies. For the purpose of the results in this paper the two possible definitions are equivalent.
} 
Assume that players can randomise. Let $S_{i}=[0,1]$ for all $i=1,2$, where $s_{i} \in S_{i}$ is to be interpreted as the probability assigned by player $i$ to action $a$. Note that the minmax payoff is 2 for each player. In the case of player 1 , it can only be obtained by $m^{1}=(a, b)$, while $m^{2}=(b, a)$ is the only action profile leading to the minmax payoff of player 2. Moreover, both $m^{1}$ and $m^{2}$ are Nash equilibria of the stage game. Finally, the mutual minmax profile is $\bar{m}=\left(m_{1}^{2}, m_{2}^{1}\right)=(b, b)$.

Now suppose that the above game is played infinitely often. If there are no bounds on the memory then the payoff of $(4,4)$ is $\mathrm{SPE}$ for any discount factor $\delta \geq 1 / 3$. To see this consider the following grim type pure strategy profile: (i) play $(a, a)$ at each date on the equilibrium path, (ii) punish deviations from $(a, a)$ by player $i=1,2$ by playing $m^{i}$ forever (once in a punishment state playing $m^{i}$ further deviations from $m^{i}$ are ignored and the play continues with $m^{i}$ ). Clearly, at each date this strategy profile induces a payoff of 4 for each player. Furthermore, the profile constitutes a SPE: First, no player wants to deviate from the equilibrium path because $4 \geq(1-\delta) 5+\delta 2$ for any $\delta \geq 1 / 3$; and second, no player wants to deviate from $m^{i}, i=1,2$, since it is a best reply.

Unfortunately, the above SPE strategy profile cannot be implemented with 1 - memory, even though the minmax action profiles for both agents are Nash equilibria of the stage game. This is because the punishment of minmaxing a deviator creates confusing instances if at each stage the players can only recall the outcome of the previous period. For example, the strategy profile is ill-defined with 1 - memory if $m^{1}=(b, a)$ is observed: it cannot be inferred if in the previous period player 1 has deviated from $(a, a)$ or if player 2 was being punished.

The impact of the 1 - memory restriction is, in fact, more profound than not being able to implement the above strategy profile. In fact, we show in this section that there does not exist any 1 - memory SPE strategy profile inducing an average payoff of $(4,4)$ for $\delta=1 / 3$. This holds even when mixed strategies are observable. In Appendix A.1 we also prove that this conclusion is robust to perturbations in the discount factor, and payoffs simultaneously. Moreover, in Appendix A.2 we generalize the result to show that the SPE payoff vector of $(4,4)$ cannot even be approximated by a 1 - memory $\varepsilon-\mathrm{CPE}$.

To establish the result of this section suppose otherwise; then there exists a 1 - memory SPE $f$ that induces an average payoff of $(4,4)$ for $\delta=1 / 3$. Since we assume mixed strategies are observable and $f$ has 1 - memory, there exist functions $g_{i}:[0,1]^{2} \rightarrow[0,1]$ for all $i=1,2$ such that $f_{i}(h)=g_{i}(T(h))$ for all $h \in H \backslash H_{0}$. 
Below we will obtain a contradiction in several steps. First, we show that the only way to obtain an average payoff of $(4,4)$ is for $f$ to play $(a, a)$ repeatedly forever, i.e. it must be that $f_{i}\left(H_{0}\right)=g_{i}(1,1)=1$ for all $i=1,2$. Let $p_{1}=f_{1}\left(H_{0}\right), q_{1}=f_{2}\left(H_{0}\right), p_{t}=g_{1}\left(p_{t-1}, q_{t-1}\right)$ and $q_{t}=g_{2}\left(p_{t-1}, q_{t-1}\right)$ for all $t \in \mathbb{N}$. Since $U_{1}(f)=U_{2}(f)=4$, it follows that

$$
8=U_{1}(f)+U_{2}(f)=\frac{2}{3} \sum_{t=1}^{\infty} \frac{8 p_{t} q_{t}+7\left(p_{t}\left(1-q_{t}\right)+q_{t}\left(1-p_{t}\right)\right)}{3^{t-1}} .
$$

Now $8 p_{t} q_{t}+7\left(p_{t}\left(1-q_{t}\right)+q_{t}\left(1-p_{t}\right)\right) \leq 8$. Therefore, condition (1) holds only if $p_{t}=q_{t}=1$ for all $t \in \mathbb{N}$. But this implies that $f_{i}\left(H_{0}\right)=g_{i}(1,1)=1$ for all $i=1,2$.

Next, we show that if player 2 were to deviate from $(a, a)$ by playing $b$, player 1 must punish by assigning a zero probability to $a$ in the period following the deviation: $g_{1}(1,0)$ must equal 0 . This is because, since player 2 can guarantee himself a payoff of 2 in every period, this deviation would at least yield him a return of $(1-\delta) 5+(1-\delta) \delta\left(4 g_{1}(1,0)+2\left(1-g_{1}(1,0)\right)\right)+2 \delta^{2}=4+4 / 9 g_{1}(1,0)$; thus, this deviation is not profitable only if $g_{1}(1,0)=0$. By a symmetric argument, $g_{2}(0,1)=0$.

When the play in period 1 is $(1,0)$, we know that in the next period player 1 must play $b$. However, this is rational only if $g_{2}(1,0)$ is high, otherwise player 1 would be tempted to play $a$ instead of playing $b$. In fact, we show next that $g_{2}(1,0)$ must be at least $1 / 6$ in order for player 1 to punish player 2 . To see this, consider for player 1 the strategy $\bar{f}_{1}$ of playing $a$ in every history: $\bar{f}_{1}(h)=1$ for all $h \in H$. Then,

$$
U_{1}\left(\bar{f}_{1}, f_{2} \mid(1,0)\right) \geq(1-\delta)\left(2 g_{2}(1,0)+2\right)+2 \delta=2+\frac{4 g_{2}(1,0)}{3} .
$$

Also, we have that

$$
U_{1}(f \mid(1,0)) \leq(1-\delta) u_{1}\left(g_{1}(1,0), g_{2}(1,0)\right)+5 \delta=(1-\delta) 5 g_{2}(1,0)+5 \delta=\frac{10 g_{2}(1,0)+5}{3}
$$

Since $f$ is a SPE, $U_{1}(f \mid(1,0)) \geq U_{1}\left(\bar{f}_{1}, f_{2} \mid(1,0)\right)$. Hence, $g_{2}(1,0) \geq 1 / 6$.

At this point the difference between the full memory and 1 - memory case is clear: In the full memory case a deviation by player 1 from $a$ leads player 2 to choose $b$ forever, while in the 1 memory case although it leads player 2 to $b$ in the first period after the deviation, in the second period after the deviation player 2 would have to play $a$ with a probability of at least $1 / 6$ if 1 were to play $a$ in the first period after the deviation. 
Consequently, the punishment with 1 - memory is less severe. This implies that a profitable deviation for player 1 exists: First player 1 chooses $b$, and then $a$ forever. We obtain the required contradiction since this deviation delivers player 1 a return of at least

$$
5(1-\delta)+2 \delta(1-\delta)+\left(4 g_{2}(1,0)+2\left(1-g_{2}(1,0)\right)\right) \delta^{2}(1-\delta)+2 \delta^{3}=4+\frac{4 g_{2}(1,0)}{27} \geq 4+\frac{2}{81}>4
$$

\subsection{Three Players}

Let $G$ be the mixed extension of the following normal form game with three players: all players have pure action spaces given by $A_{i}=\{a, b\}$,

$$
u_{3}\left(a_{1}, a_{2}, a_{3}\right)= \begin{cases}4 & \text { if } a_{3}=a \text { and } \\ 2 & \text { if } a_{3}=b .\end{cases}
$$

for all $a_{1} \in A_{1}$ and $a_{2} \in A_{2}, u_{1}$ and $u_{2}$ are defined by Table 1 above if $a_{3}=a$ and arbitrarily if $a_{3}=b$.

Clearly, $a$ is a strictly dominant strategy for player 3. Therefore, if $f$ is a SPE, then $f_{3}(h)=a$ for all $h \in H$; thus, we are effectively in the same situation as in the above subsection. Therefore, arguing as in the previous section, one can show that $(4,4,4)$ is a SPE payoff that cannot be supported by a $1-$ memory $\mathrm{SPE}$ for $\delta=1 / 3$. Moreover, by the same arguments as in Appendix A.1, this conclusion is robust to perturbations in the discount factor, and payoffs simultaneously.

\section{Confusion-Proof Paths and 1 - Memory}

Following Abreu (1988), $f \in F$ is a simple strategy profile represented by $n+1$ paths $\left(\pi^{(0)}, \pi^{(1)}, \ldots\right.$, $\left.\pi^{(n)}\right)$ if $f$ specifies: (i) play $\pi^{(0)}$ until some player deviates singly from $\pi^{(0)}$; (ii) for any $j \in N$, play $\pi^{(j)}$ if the $j$ th player deviates singly from $\pi^{(i)}, i=0,1, \ldots, n$, where $\pi^{(i)}$ is the ongoing previously specified path; (iii) continue with the ongoing specified path $\pi^{(i)}, i=0,1, \ldots, n$, if no deviations occur or if two or more players deviate simultaneously. These strategies are simple because the play of the game is always in only $(n+1)$ states, namely, in state $j \in\{0, \ldots, n\}$ where $\pi^{(j), t}$ is played, for some $t \in \mathbb{N}$. In this case, we say that the play is in phase $t$ of state $j$. A profile $\left(\pi^{(0)}, \pi^{(1)}, \ldots, \pi^{(n)}\right)$ of $n+1$ outcome paths is subgame perfect if the simple strategy represented by it is a SPE . 
Henceforth, when the meaning is clear, we shall use the term $\left(\pi^{(0)}, \pi^{(1)}, \ldots, \pi^{(n)}\right)$ to refer to both an $n+1$ outcome paths as well as to the simple strategy profile represented by these paths. Also, when referring to a profile of $n+1$ outcome paths, we shall not always explicitly mention $n+1$ and simply refer to it by a profile of outcome paths.

Abreu (1988) used the concept of simple strategies to characterize the set of subgame perfect equilibria. In this section, we consider simple strategy profiles that can be implemented with 1 memory. For this purpose, we introduce the notion of a confusion-proof profile of outcome paths and show in Proposition 1 below that that a profile of outcome paths can be supported by a 1 memory simple strategy if and only if it is confusion proof. The construction used in Proposition 1 is our main tool and is used throughout the paper.

The notion of a confusion-proof profile of outcome paths is motivated by the following observations. For a profile of simple strategies to be supported by a 1 - memory simple strategy, players need to find out the correct state of the play by only observing the action profile in the previous period. This clearly is not always possible. To see this consider a simple strategy represented by the profile of paths $\left(\pi^{(0)}, \pi^{(1)}, \ldots, \pi^{(n)}\right)$. Then, three kinds of complications can arise if the strategies have 1 - memory.

The first kind of complication happens when

$$
\pi^{(i), t}=\pi^{(j), r} \text { for some } i, j \in\{0,1, \ldots, n\} \text { and } t, r \in \mathbb{N} \text {. }
$$

That is, the action profile in phase $t$ of state $i$ is the same as that in phase $r$ of state $j$. Since players condition their behavior only last period's action profile, the players cannot distinguish between phase $t$ of state $i$ and phase $r$ of state $j$, and therefore the simple strategy cannot be implemented, unless $\pi^{(i), t+1}=\pi^{(j), r+1}$.

The second kind of complication arises when

$$
\pi_{-k}^{(i), t}=\pi_{-k}^{(j), r} \text { for some } i, j \in\{0,1, \ldots, n\}, k \in N \text { and } t, r \in \mathbb{N} \text {. }
$$

In words, every player other than $k \in N$ takes the same action in phase $t$ of state $i$ and in phase $r$ of state $j$. Then if, for example, the last period's action profile is $\pi^{(j), r}$, the players would not be able to deduce whether the play in the previous period was in phase $t$ of state $i$ and player $k$ deviated to $\pi_{k}^{(j), r}$ or whether it was in phase $r$ of state $j$ and no deviation occur. Since a deviation by player 
$k$ from $\pi_{k}^{(i), t}$ to $\pi_{k}^{(j), r}$ in phase $t$ of state $i$ is impossible to be detected by observing only the action in the last period, the simple strategy cannot be implemented, unless $\pi^{(i), t+1}=\pi^{(j), r+1}=\pi^{(k), 1}$.

The third kind of complication appears when

$$
\pi_{-l, m}^{(i), t}=\pi_{-l, m}^{(j), r} \text { for some } i, j \in\{0,1, \ldots, n\}, l, m \in N \text { and } t, r \in \mathbb{N}
$$

In words, all players other than $l$ and $m \in N$ take the same action both in phase $t$ of state $i$, and in phase $r$ of state $j$. Then, if the last period's action profile is given by $\left(\pi_{l}^{(i), t}, \pi_{m}^{(j), r},\left(\pi_{k}^{(i), t}\right)_{k \neq l, m}\right)=$ $\left(\pi_{l}^{(i), t}, \pi_{m}^{(j), r},\left(\pi_{k}^{(j), r}\right)_{k \neq l, m}\right)$, players, looking back one period, can conclude that either player $l$ or player $m$ has deviated. But, they cannot be certain of the identity of the deviator. Consequently, both of them must be punished. This requires $\pi^{(l)}=\pi^{(m)}$.

These observations are formalized below as follows. For any profile of outcome paths $\left(\pi^{(0)}, \pi^{(1)}\right.$, $\left.\ldots, \pi^{(n)}\right) \subseteq \Pi^{n+1}$, let

$$
\Omega(\{i, t\},\{j, r\})=\left\{k \in N: \pi_{k}^{(i), t} \neq \pi_{k}^{(j), r}\right\}
$$

be the set of players whose actions in phase $t$ of stage $i$ and in phase $r$ stage $j$ are different.

Definition 2 A profile $\left(\pi^{(0)}, \ldots, \pi^{(n)}\right) \in \Pi^{n+1}$ of outcome paths is confusion-proof if for any $i, j \in$ $\{0,1, \ldots, n\}$ and $t, r \in \mathbb{N}$ the following holds:

1. If $\Omega(\{i, t\},\{j, r\})=\emptyset$, then $\pi^{(i), t+1}=\pi^{(j), r+1}$.

2. If $\Omega(\{i, t\},\{j, r\})=\{k\}$ for some $k \in N$, then $\pi^{(i), t+1}=\pi^{(j), r+1}=\pi^{(k), 1}$.

3. If $\Omega(\{i, t\},\{j, r\})=\{k, l\}$ for some $k$ and $l \in N$, then $\pi^{(k)}=\pi^{(l)}$.

The above observations, which motivated the definition of confusion-proof outcome paths, suggest that confusion-proofness is necessary to support a profile of outcome paths with an 1 - memory simple strategy. The next Proposition asserts that confusion-proofness is, in fact, not only a necessary but also a sufficient condition to support a profile of outcome paths with 1 - memory.

Proposition 1 A profile of outcome paths is confusion-proof if and only if there exists a 1 -memory simple strategy represented by it. 
The 1 - memory strategy $f$ supporting the confusion-proof profile of outcome paths $\left(\pi^{(i)}\right)_{i=0,1, \ldots, n}$ is as follows: If the last period of a given history equals $\pi^{(j), t}$, for some $j=0,1, \ldots, n$ and $t \in \mathbb{N}$, then player $i$ chooses $\pi_{i}^{(j), t+1}$. If only player $k \in N$ deviated from the outcome $\pi^{(j)}$ in the last period of the history, then player $i$ chooses $\pi_{i}^{(k), 1}$. Finally, if more then one player deviated from the outcome $\pi^{(j)}$ in the last period of the history, then player $i$ chooses $\pi_{i}^{(j), t+1}$ (thus deviations involving more than one player are ignored). Since $f$ has 1 - memory and has the structure of a simple strategy, we say that $f$ is a 1 - memory simple strategy. As before, the profile $\left(\pi^{(0)}, \ldots, \pi^{(n)}\right)$ represents $f$. The main task of the sufficiency part of the proof of Proposition 1 is to show that $f$ is well defined, which we show follows from $\left(\pi^{(0)}, \ldots, \pi^{(n)}\right)$ being confusion-proof.

Before turning to the equilibrium characterization with 1 - memory, we shall next provide a set of easily tractable sufficient conditions for a profile of outcome paths to be confusion-proof.

Lemma 1 A profile of outcome paths $\left(\pi^{(0)}, \pi^{(1)}, \ldots, \pi^{(n)}\right)$ is confusion-proof if one of the following conditions hold:

1. If $n \geq 3$, then for all $i, j \in\{0,1, \ldots, n\}$ and $t, r \in \mathbb{N}$ satisfying $(i, t) \neq(j, r)$ the number of players whose actions in phase $t$ of stage $i$ and in phase $r$ stage $j$ are different is at least three:

$$
|\Omega(\{i, t\},\{j, r\})| \geq 3
$$

2. If $n=2$, then

(a) players have the same punishment path:

$$
\pi^{(1)}=\pi^{(2)}
$$

(b) for all $i, j \in\{0,1,2\}$ and $t, r \in \mathbb{N}$ satisfying $(i, t) \neq(j, r)$ and $i=1 \Rightarrow j \neq 2$ the actions of each agent is distinct:

$$
\pi_{l}^{(i), t} \neq \pi_{l}^{(j), r} \text { for any } l=1,2
$$

The condition of the above Lemma for the case of three or more players is clearly sufficient for confusion-proofness. Similarly, if $\left(\pi^{(0)}, \pi^{(1)}, \pi^{(2)}\right)$ satisfies $(6)$ and (7) in a game with two players, then for all $i, j \in\{0,1,2\}$ and $t, r \in \mathbb{N}$ such that $(i, t) \neq(j, r)$, it follows that $|\Omega(\{i, t\},\{j, r\})|=2$, 
except when $i, j \in\{1,2\}$. This together with (6) imply that $\left(\pi^{(0)}, \pi^{(1)}, \pi^{(2)}\right)$ is confusion-proof when $n=2$.

Conditions (5) and (7), however, are not necessary for confusion-proofness in the case when $n>2$ and $n=2$, respectively. For instance, $\left(\pi^{(0)}, \ldots, \pi^{(n)}\right)$ defined by $\pi^{(j), t}=s \in S$ for all $j \in\{0, \ldots, n\}$ and $t \in \mathbb{N}$ is confusion-proof but it does not satisfy these conditions. Also condition (6) is not necessary for confusion-proofness. To see that, let $\left(\pi^{(0)}, \pi^{(1)}, \pi^{(2)}\right)$ be defined by $\pi^{(j), t}=s \in S$ for all $j \in\{0,1\}$ and $t \in \mathbb{N}$, and by

$$
\pi^{(2), t}= \begin{cases}\left(\bar{s}_{1}, s_{2}\right) & \text { if } t=1, \\ s & \text { if } t \geq 2 .\end{cases}
$$

Then, $\left(\pi^{(0)}, \pi^{(1)}, \pi^{(2)}\right)$ is confusion-proof but $\pi^{(1)} \neq \pi^{(2)}$. However, as the following remark demonstrates, with $n=2$ the above example is the only possible confusion proof paths that violates condition (6) and therefore identical punishment paths for both players is almost necessary for confusion proofness in 2 - player games.

Remark 1 If $n=2$ and $\left(\pi^{(0)}, \pi^{(1)}, \pi^{(2)}\right)$ is confusion-proof, then either $\pi^{(1)}=\pi^{(2)}$ or there exists $i \in N, s=\left(s_{i}, s_{-i}\right) \in S$ and $\bar{s}_{i} \in S_{i}$ such that $\pi^{(i), t}=s$ for all $t \in \mathbb{N}$ and for $j \in N, j \neq i$

$$
\pi^{(j), t}= \begin{cases}\left(\bar{s}_{i}, s_{-i}\right) & \text { if } t=1, \\ s & \text { if } t \geq 2 .\end{cases}
$$

As it clear from the above, the analysis of the confusion proof simple paths, and hence 1 memory strategies, is considerably different for the case of 2 - player games from that with three or more players. The basic difference between two cases is similar to that found in the implementation literature. Here, as in there, when there are only two players, it may not be possible to detect which of the two players have deviated and as a result both must be punished with the same punishment path (condition (6)), whenever a deviation is detected.

\section{$5 \quad$ Discounting}

In this section, we assume that all agents discount the future returns by a common discount factor $\delta \in(0,1)$. Thus the payoff in the supergame $G^{\infty}(\delta)$ of $G$ is now given by

$$
U_{i}(f)=(1-\delta) \sum_{t=1}^{\infty} \delta^{t-1} u_{i}\left(\pi^{t}(f)\right)
$$


Also, for any $\pi \in \Pi, t \in \mathbb{N}$, and $i \in N$, let $V_{i}^{t}(\pi)=(1-\delta) \sum_{r=t}^{\infty} \delta^{r-t} u^{i}\left(\pi^{r}\right)$ be the continuation payoff of player $i$ at date $t$ if the outcome path $\pi$ is played. For simplicity, we write $V_{i}(\pi)$ instead of $V_{i}^{1}(\pi)$.

An outcome path $\pi$ is a subgame perfect outcome path if there exists a SPE $f$ such that $\pi=\pi(f)$.

A profile of outcome paths $\left(\pi^{(0)}, \ldots, \pi^{(n)}\right) \in \Pi^{n+1}$ is weakly enforceable if

$$
V_{i}^{t}\left(\pi^{(j)}\right) \geq(1-\delta) \max _{s_{i}} u_{i}\left(s_{i}, \pi_{-i}^{(j), t}\right)+\delta V_{i}\left(\pi^{(i)}\right)
$$

for all $i \in N, j \in\{0,1, \ldots, n\}$ and $t \in \mathbb{N}$.

From Abreu (1988), it is well known that weak enforceability is equivalent to subgame perfection. More precisely, an outcome path $\pi^{(0)}$ is a SPE outcome path if and only if there exists a weakly enforceable profile of outcome paths $\left(\pi^{(0)}, \ldots, \pi^{(n)}\right)$.

In our setting we note that, by Proposition 1, any weakly enforceable, confusion-proof profile of outcome paths can be supported by a 1 - memory simple SPE strategy. In particular, the same holds for any SPE payoff vector that can be obtained by a confusion-proof profile of outcome paths. Moreover, it is worthwhile to note that connectedness of strategy spaces is not needed for these conclusions that are summarized in the following corollary to Proposition 1.

Corollary 1 Let u be SPE payoff vector that can be supported by a weakly enforceable, confusionproof profile of outcome paths. Then, there is a 1 - memory SPE strategy $f$ such that $U(f)=u$.

In general, as was shown by the examples in Section 3, we cannot support all subgame perfect payoff vectors by 1 - memory SPE strategies. In fact, the best that can be hoped for is to obtain them approximately. There are three aspects involved in our approximations. The first involves the equilibrium concept in question. To that regard, we employ the notion of contemporaneous $\varepsilon$ - perfect equilibrium $(\varepsilon-\mathrm{CPE})$ that is formally defined as follows (see Mailath, Postlewaite, and Samuelson (2005)): For all $\varepsilon \geq 0$, a strategy profile $f \in F$ is a contemporaneous $\varepsilon$ - Nash equilibrium of the supergame of $G$ if for all $i \in N, V_{i}^{t}(\pi(f)) \geq V_{i}^{t}\left(\pi\left(\hat{f}_{i}, f_{-i}\right)\right)-\varepsilon$ for all $t \in \mathbb{N}$ and $\hat{f}_{i} \in F_{i}$. A strategy vector $f \in F$ is a contemporaneous $\varepsilon$ - perfect equilibrium of the supergame of $G$ if $f \mid h$ is a contemporaneous $\varepsilon-$ Nash equilibrium for every $h \in H$.

The second kind of approximation features the distance in the payoff space: given a SPE payoff vector, can a payoff vector close to it be sustained with a 1 - memory SPE? 
The third approximation concerns the discount factor. With no memory restrictions, the Folk Theorem states that any strictly individual rational payoff be supported in SPE for $\delta$ sufficiently large. Does the same hold with 1 - memory?

Since any SPE has to be weakly enforceable, it turns out that a slack in the incentive equations (8) is needed in order to perform the required approximations. This leads us to introduce the notion $\varepsilon-$ strictly enforceability of a SPE payoff vector.

Definition 3 For all $\varepsilon \geq 0$, a payoff vector $u \in \mathbb{R}^{n}$ is a $\varepsilon$-strictly enforceable SPE payoff if there exists a profile of $n+1$ outcome paths $\left(\hat{\pi}^{(0)}, \ldots, \hat{\pi}^{(n)}\right)$ such that $V_{i}\left(\hat{\pi}^{(0)}\right)=u_{i}$ and

$$
\inf _{t \in \mathbb{N}}\left(V_{i}^{t}\left(\hat{\pi}^{(j)}\right)-\left((1-\delta) \max _{s_{i}} u_{i}\left(s_{i}, \hat{\pi}_{-i}^{(j), t}\right)+\delta V_{i}\left(\hat{\pi}^{(i)}\right)\right)\right)>-\varepsilon
$$

for all $i \in N$ and all $j \in\{0,1, \ldots, n\}$.

Thus, an equilibrium simple strategy profile that induces an $\varepsilon$-strictly enforceable SPE payoff is such that at any date and at any history, the maximum gain a player can make from one-period deviation from the equilibrium strategy is less than $\varepsilon$.

For convenience, if $u$ is an $\varepsilon$ - strictly enforceable SPE payoff with $\varepsilon=0$, we simply say that $u$ is a strictly enforceable SPE payoff.

Theorem 1 demonstrates that under certain conditions, any $\varepsilon$-strictly enforceable SPE payoff can be approximately supported with a $1-$ memory $\varepsilon-\mathrm{CPE}$.

Theorem 1 Let $\varepsilon \geq 0$ and $u$ be an $\varepsilon$ - strictly enforceable SPE payoff vector induced by the simple strategy profile $\left(\hat{\pi}^{(0)}, \ldots, \hat{\pi}^{(n)}\right)$. Then, for every $\eta>0$ there is a 1 - memory $\varepsilon-C P E$ strategy profile $f$ such that $|U(f)-u|<\eta$, provided that either $n \geq 3$, or $n=2$ and $\hat{\pi}^{(1)}=\hat{\pi}^{(2)}$.

Remark 2 Note that when $\varepsilon=0$, Theorem 1 shows that every neighborhood of any strictly enforceable SPE payoff profile contains a payoff profile that can be obtained with a 1 - memory SPE if either $n \geq 3$, or $n=2$ and $\hat{\pi}^{(1)}=\hat{\pi}^{(2)}$. Thus, in these cases, we can approximate a payoff profile using subgame perfection with 1 - memory, if players strictly prefer to follow the associated simple strategy at every subgame.

Although there are SPE payoff vectors which are not strictly enforceable (not satisfying Definition 3 for $\varepsilon=0$ ), note that any SPE payoff profile is weakly enforceable, and hence is $\varepsilon$-strictly enforceable for all $\varepsilon>0$. This simple observation implies the following corollary to Theorem 1 . 
Corollary 2 For every SPE payoff vector $u \in \mathbb{R}^{n}$, and every $\eta>0$, there is a 1 - memory $\eta$ CPE strategy profile $f$ such that $|U(f)-u|<\eta$, whenever either $n \geq 3$, or $n=2$ and there exist a SPE simple strategy described by $\left(\hat{\pi}^{(0)}, \hat{\pi}^{(1)}, \hat{\pi}^{(2)}\right)$ such that $\hat{\pi}^{(1)}=\hat{\pi}^{(2)}$ and $V_{i}\left(\hat{\pi}^{(0)}\right)=u_{i}$ for all $i \in N$.

This corollary to Theorem 1 is in the same spirit as Theorem 4.1 of Kalai and Stanford (1988). It shows that with three more players, given any $\eta>0$, every SPE payoff vector can be approximately obtained by a 1 - memory $\eta$ - CPE. In other words, the value of any recall beyond observing the last period, is arbitrarily small. Moreover, the same conclusion holds for 2 - player games if the punishment paths needed to enforce the original equilibrium are the same for both players.

The proof of Theorem 1 involves showing that under the assumptions of the Theorem, together with $S_{i}$ being connected and $u_{i}$ being continuous, the given profile of outcome paths $\left(\hat{\pi}^{(0)}, \ldots, \hat{\pi}^{(n)}\right)$ supporting $u$ as an $\varepsilon$ - strictly enforceable SPE payoff vector can be approximated by a confusionproof profile of outcome paths $\left(\bar{\pi}^{(0)}, \ldots, \bar{\pi}^{(n)}\right)$, that is arbitrarily close to the first in terms of the distance in payoffs. This implies that the latter profile of outcome paths is also an $\varepsilon-$ strictly enforceable SPE. Applying Proposition 1 completes the proof.

Theorem 1 establishes that any strictly enforceable utility vector can be approximated by a 1 - memory SPE payoff vector. Can such 1 - memory implementation of strictly enforceable utility be exact? Clearly, if a strictly enforceable utility vector $u$ has the additional property that it can be obtained by a confusion proof profile of outcome paths, $\left(\hat{\pi}^{(0)}, \ldots, \hat{\pi}^{(n)}\right)$, then there exists a 1 - memory simple strategy profile that supports $u$ exactly. Even if $\left(\hat{\pi}^{(0)}, \ldots, \hat{\pi}^{(n)}\right)$ is not confusion-proof, but is strictly enforceable and the single path $\hat{\pi}^{(0)}$ is implementable with 1 - memory $\left(\hat{\pi}^{(0)}\right.$ does not involve any confusing instances), $u$ can still be sustained exactly by a 1 - memory SPE. As in the proof of Theorem 1, this can be established by constructing another punishment profile $\left(\bar{\pi}^{(1)}, \ldots, \bar{\pi}^{(n)}\right)$ such that $\left(\hat{\pi}^{(0)}, \bar{\pi}^{(1)}, \ldots, \bar{\pi}^{(n)}\right)$ is confusion-proof simple equilibrium and hence 1 - memory implementable. ${ }^{9}$ To show this formally, we shall next define a confusion-proof single outcome path and a confusion-proof payoff vector.

A single outcome path $\pi$ is free of confusion if it satisfies the following three conditions. First, if the vector of actions is the same in two different periods, then the action profile in the period following one of those periods must equal the action profile following the other period. Second, it

\footnotetext{
${ }^{9}$ In the next section, we shall use this observation to establish our Folk Theorem type results with 1 - memory.
} 
cannot be the case that there is exactly one player whose action is different in two given periods. And third, with more than two players, it cannot be the case that there is exactly two players whose actions are different in two given periods. These restrictions are described in Definition 4 below, and are similar to those made in Definition 2. As before, the set of players that in a given outcome path play a different actions in different periods is a key concept. Formally, for any outcome paths $\pi \in \Pi$ this set is defined by

$$
\Omega(t, r)=\left\{i \in N: \pi_{i}^{t} \neq \pi_{i}^{r}\right\} \text { for any two periods } t \text { and } r \in \mathbb{N} \text {. }
$$

Definition 4 A single path $\pi \in \Pi$ is confusion-proof if the following properties hold:

1. $|\Omega(t, r)|=0$ for some $t, r \in \mathbb{N}$, implies $\pi^{t+1}=\pi^{r+1}$.

2. There are no $t, r \in \mathbb{N}$ with $|\Omega(t, r)|=1$.

3. If $n>2$, then $|\Omega(t, r)| \neq 2$ for all $t, r \in \mathbb{N}$.

We define the set of $\Pi^{c p}$ to be the set of all single confusion-proof paths. Furthermore, a payoff vector $u$ is confusion-proof if it can supported by a single confusion-proof path: there exists $\pi \in \Pi^{c p}$ such that $V(\pi)=u$.

Using the same techniques as in the proof of Theorem 1 one can prove the following Proposition.

Proposition 2 Suppose that $u$ is supported by a single confusion-proof path $\pi^{(0)} \in \Pi^{c p}$. Assume also that there exists $n$ paths $\left(\pi^{(1)}, \ldots, \pi^{(n)}\right) \in \Pi^{n}$ such that $\left(\pi^{(0)}, \pi^{(1)}, \ldots, \pi^{(n)}\right)$ is strictly enforceable. Then, there is a 1 - memory SPE strategy $f$ such that $U(f)=u$, provided that either $n \geq 3$, or $n=2$ and $\pi^{(1)}=\pi^{(2)}$.

Thus, confusion-proof strictly enforceable SPE payoffs are most well-suited for our goal of supporting payoff vectors by 1 - memory SPE strategies. This makes it natural to ask whether we can describe the set of such payoffs. In the next section, we will provide a partial description of that set, for all sufficiently large discount factors.

Before considering the case of patient players, we would like to discuss the relation between the examples presented in Section 3 and the results in this section. In the 3 -player example, recall that we identified for $\delta=1 / 3$ a SPE (and Pareto optimal) payoff vector $(4,4,4)$ which cannot be 
supported by any 1 - memory equilibrium (the result was also robust to small perturbations of stage game payoffs and/or $\delta$ ). On the other hand, Corollary 2 displays that the same payoff vector can arbitrarily closely be approximated with a $1-$ memory $\varepsilon-\mathrm{CPE}$, for all $\varepsilon>0$. Indeed, it is not very difficult to show that a stronger result holds: since this payoff can be supported by a confusion-proof single path (repeating $(a, a, a)$ forever), it can be obtained exactly in $\varepsilon$ - CPE with 1 - memory, for all $\varepsilon>0$. Therefore, these observations imply that there is a discontinuity in the following sense: even though the SPE payoff vector $(4,4,4)$ can be obtained with a $1-$ memory $\varepsilon-\mathrm{CPE}$ for all $\varepsilon>0$, it cannot be exactly sustained with 1 - memory SPE (the same discontinuity holds if we perturb the stage game payoffs and/or $\delta$ ). To see the nature of this discontinuity, note that the payoff profile $(4,4,4)$ cannot be obtained with a strictly enforceable simple strategy because player 3 has a dominant strategy that induces a payoff of 4 at every stage game (therefore, the continuation payoff of player 3 is the same at every history). This implies that the hypothesis of Theorem 1 (and Proposition 2) on strict enforceability does not hold for $(4,4,4)$ when $\varepsilon=0$. On the other hand, when $\varepsilon$ exceeds zero the payoff at different histories do not have to be the same, and as a result, $(4,4,4)$ can be obtained as an $\varepsilon$ - strictly enforceable simple strategy for $\varepsilon>0$; thus in the case of the example the hypothesis of Theorem 1 holds when $\varepsilon>0$.

Considering the 2 - player example of Section 3 , recall that when $\delta=1 / 3$ not only we identified a SPE (and Pareto optimal) payoff vector $(4,4)$ which cannot be supported by any 1 - memory SPE (the result is also robust to small perturbations of payoffs and/or $\delta$ ), we also showed that $(4,4)$ cannot even be approximated by a 1 - memory $\varepsilon-\mathrm{CPE}$, for small $\varepsilon>0 .{ }^{10}$ Therefore, the conclusions of the all the main results in this section (Theorem 1, Remark 5, Corollary 2 and Proposition 2) do not hold for the payoff vector $(4,4)$ in our 2 - player example. The reason for this is that for the 2 - player case all the results in this section require a common punishment path; whereas to enforce the payoff vector $(4,4)$ (or a payoff close to it) as a SPE in the example requires different punishment paths for the two players. ${ }^{11}$

\footnotetext{
${ }^{10}$ Thus, in this example there is not even a discontinuity with respect to the $\varepsilon-\mathrm{CPE}$.

${ }^{11}$ Note that the payoff vector $(4,4)$ in the example when $\delta=1 / 3$ is not a strictly enforceable SPE. This implies that this payoff vector is inconsistent with the hypothesis of Theorem 1, Remark 5 and Proposition 2 for another reason other than the lack of common punishment paths. However, since the conclusion of the example is robust to small perturbations of the payoffs, it follows that we can always construct another example in which a strictly enforceable with different punishment paths cannot be implemented by a 1 - memory SPE, thus, highlighting that
} 


\section{$6 \quad$ Folk Theorems}

\subsection{Folk Theorem with Discounting}

Let $D=\operatorname{co}(u(S)), \mathcal{U}=\left\{y \in D: y_{i} \geq v_{i}\right.$ for all $\left.i \in N\right\}$ and $\mathcal{U}^{0}=\left\{y \in D: y_{i}>v_{i}\right.$ for all $\left.i \in N\right\}$. The set $\mathcal{U}$ (resp. $\mathcal{U}^{0}$ ) is the set of (resp. strictly) individually rational payoffs.

Fudenberg and Maskin (1986)'s Folk Theorem for repeated games with discounting establishes that any strictly individually rational payoff profiles $u \in \mathcal{U}^{0}$ can be sustained as a SPE payoff profile if the players are sufficiently patient and $\mathcal{U}$ has full-dimension $(\operatorname{dim}(\mathcal{U})=n)$. However, the discussion in the previous section shows that a payoff profile $u \in D$ can be sustained as a SPE with 1 - memory only if there exists a confusion proof single path that induces $u{ }^{12}$ Our first result in this section shows that if players are sufficiently patient then all $u \in \mathcal{U}^{0}$ with this property can be implemented exactly by a 1 - memory SPE if $u$ is bounded away from the boundary of $\mathcal{U}^{0}$ and the full-dimensionality condition holds. Using this result, we shall then show that all $u \in \mathcal{U}$ can be implemented approximately by a 1 - memory SPE.

Fix any discount factor $\delta<1$. Then for any $\alpha \in \mathcal{U}^{0}$ let $\Lambda(\alpha, \delta) \subseteq \Pi$ be the set of all single confusion-proof outcome paths $\pi \in \Pi^{c p}$ such that the continuation payoff of every player $i$ at each date $t$ if $\pi$ is played is no less than $\alpha_{i}:{ }^{13}$

$$
\Lambda(\alpha, \delta)=\left\{\pi \in \Pi^{c p}: V_{i}^{t}(\pi) \geq \alpha_{i} \text { for all } i \in N \text { and } t \in \mathbb{N}\right\}
$$

Also, denote the set of confusion-proof payoff vectors that are supported by the set $\Lambda(\alpha, \delta)$ by

$$
C(\alpha, \delta)=\left\{u \in \mathbb{R}^{n}: u=V(\pi) \text { for some } \pi \in \Lambda(\alpha, \delta)\right\}
$$

The next Proposition shows that all payoffs in $C(\alpha, \delta)$ can be supported by 1 - memory SPE if $\delta$ is sufficiently high.

Proposition 3 Suppose that either $\operatorname{dim}(\mathcal{U})=n$ or $n=2$ and $\mathcal{U}^{0} \neq \emptyset$. Then for all $\alpha \in \mathcal{U}^{0}$, there exists $\bar{\delta} \in(0,1)$ such that for all $\delta \geq \bar{\delta}$ the following holds: for all payoffs $u \in C(\alpha, \delta)$ there exists a 1 - memory SPE strategy $f$ with $U(f)=u$.

the critical factor in the example is the lack of common punishment paths.

${ }^{12}$ This property clearly holds for all $u \in D$ if the payoff space $u(S)$ were convex. In this case $D=u(S)$ and therefore any payoff vector in $D$ can be obtained by the repetition of the same action profile.

${ }^{13}$ Note that $V_{i}^{t}(\pi)$ depends on the discount factor $\delta$ but for ease of exposition we shall not make this explicit. 
Since any payoff $u \in C(\alpha, \delta)$ can be sustained by a confusion proof single path it follows, by Proposition 2, that to prove Proposition 3 it is sufficient to show that for sufficiently high discount factor and any $u \in C(\alpha, \delta)$ there exists a strictly enforceable simple strategy profile given by $\left(\pi^{(0)}, \pi^{(1)}, \ldots, \pi^{(n)}\right)$ such that $V\left(\pi^{(0)}\right)=u$, and furthermore, in the 2 - player case the punishment paths are the same.

If the payoff space $u(S)$ were convex (e.g. if correlated strategies were allowed), demonstrating the existence of such a strictly enforceable simple strategy profile would be completely standard and would follow in a relatively straightforward manner using the method developed in Fudenberg and Maskin (1986) (in this case, as we mentioned in Footnote 6.1, any payoff vector $u \in D$ can be obtained by the repetition of the same actions, and therefore can be trivially sustained by a confusion-proof single path).

However, in our set-up $u(S)$ is not necessarily convex. This complicates the construction of the required simple strategy profile $\left(\pi^{(0)}, \pi^{(1)}, \ldots, \pi^{(n)}\right)$ in the proof of Proposition 3 . In particular, to prove the result with more than two players, we construct, for each player $i \in N$, a punishment path $\pi^{(i)}$ that minmaxes $i$ (plays $m^{i}$ ) for $T$ periods and then plays another path $\widehat{\pi}^{(i)}$. The path $\widehat{\pi}^{(i)}$ is chosen so that its payoff $V_{i}\left(\widehat{\pi}^{(i)}\right)$ for player $i$ to have the following four properties. First, it is strictly below the payoff $i$ receives on the equilibrium path at any date (i.e., $\left.V_{i}\left(\widehat{\pi}^{(i)}\right)<\inf _{t} V_{i}^{t}\left(\pi^{(0)}\right)\right)$. Second, it strictly exceeds the minmax payoff for $i$. Third, it is below its continuation payoff at any date (i.e., $V_{i}\left(\widehat{\pi}^{(i)}\right) \leq V_{i}^{t}\left(\widehat{\pi}^{(i)}\right)$ for all $\left.t \in \mathbb{N}\right)$. Fourth, it is below the payoff obtained by punishing any other player at any date (i.e., $V_{i}\left(\widehat{\pi}^{(i)}\right) \leq V_{i}^{t}\left(\widehat{\pi}^{(j)}\right)$ for all $j \in N \backslash\{i\}$ and all $t \in \mathbb{N}$ ). All these properties are intuitive. The first guarantees that a player that deviates from the equilibrium payoff is punished regardless of the date of the deviation. The second and the third display the typical "stick and carrot" nature of the punishments: players are punished more severely early on. Finally, the fourth properties gives each player an incentive to punish deviators. Ensuring these properties without assuming correlated strategies (or more generally without assuming $u(S)$ is convex) is complicated because each path $\widehat{\pi}^{(i)}$ may consist of playing a finite sequence of action profiles repeatedly; as a result $V_{i}^{t}\left(\widehat{\pi}^{(i)}\right)$ may not equal $V_{i}^{t^{\prime}}\left(\widehat{\pi}^{(i)}\right)$ for any two dates $t$ and $t^{\prime}$. With correlated strategies the path $\widehat{\pi}^{(i)}$ could be constructed in such a way such that it involves playing a single action profile repeatedly and therefore $V_{i}^{t}\left(\widehat{\pi}^{(i)}\right)=V_{i}^{t^{\prime}}\left(\widehat{\pi}^{(i)}\right)$ for all $t$ and $t^{\prime}$. Therefore, the above four properties are easier to satisfy in the latter case than in the former (in fact, the third 
property is automatically satisfied in the latter case because since $V_{i}^{t}\left(\widehat{\pi}^{(i)}\right)=V_{i}^{t^{\prime}}\left(\widehat{\pi}^{(i)}\right)$ for all $t$ and $\left.t^{\prime}\right)$.

In the case of two players, the structure of the proof is the same. However, since the punishment path needs to be common to both players, it is considerably more difficult task to construct a common punishment path with the above four properties. In addition, as in Fudenberg and Maskin (1986), we need to use a mutual minmax action in the initial phase of the punishment path.

Proposition 3 shows that for all $\alpha \in \mathcal{U}^{0}, C(\alpha, \delta)$ is contained in the set of payoffs supported by 1 - memory SPE strategies for large $\delta$. We shall now use this result to establish a 1 - memory Folk Theorem result for the set of individually rational payoffs $\mathcal{U}$. This is obtained by first establishing that any $u \in \mathcal{U}$ can be approximated with a confusion-proof payoff profile in $C(\alpha, \delta)$ if $\delta$ is sufficiently close to one.

Lemma 2 Suppose that either $\operatorname{dim}(\mathcal{U})=n$ or $n=2$ and $\mathcal{U}^{0} \neq \emptyset$. For all $u \in \mathcal{U}$ and $\zeta>0$ there exists $\alpha \in \mathcal{U}^{0}$ and $\tilde{\delta} \in(0,1)$ such that for all $\delta \geq \tilde{\delta}$ there is $\tilde{u} \in C(\alpha, \delta)$ with $\|u-\tilde{u}\|<\zeta$.

Combining Proposition 3 and Lemma 2 we obtain the perfect 1 - memory Folk Theorem.

Theorem 2 Suppose that either $\operatorname{dim}(\mathcal{U})=n$ or $n=2$ and $\mathcal{U}^{0} \neq \emptyset$. Then, for all $u \in \mathcal{U}$ and $\zeta>0$, there exists $\delta^{*} \in(0,1)$ such that for all $\delta \geq \delta^{*}$, there is a 1 - memory SPE strategy $f$ with $\|U(f)-u\|<\zeta$.

\subsection{No Discounting}

In this section we assume that players do not discount the future and are interested in the long-term average payoff. The payoff in the supergame $G^{\infty}(1)$ of $G$ is now given by:

$$
U_{i}^{\infty}(f)=\liminf _{T \rightarrow \infty} \frac{1}{T} \sum_{t=1}^{T} u_{i}\left(\pi^{k}(f)\right) .
$$

For all $\pi \in \Pi$ and $i \in N$, we let $V_{i}^{\infty}(\pi)=\liminf _{T \rightarrow \infty} \frac{1}{T} \sum_{t=1}^{T} u_{i}\left(\pi^{t}\right)$ be the supergame payoff of player $i$ when the path $\pi$ is implemented. Finally, denote the set of confusion-proof payoffs in the supergame $G^{\infty}(1)$ that can be obtained through the repetition of a cycle by

$$
C=\left\{u \in \mathbb{R}^{n}: u=V_{i}^{\infty}(\pi) \text { for some } \pi \in \Pi^{c p} \text { that consists of a repetition of a cycle }\right\}
$$


We shall now establish a similar Folk Theorem result for the no discounting case to that in the previous section without assuming the full-dimensionality condition. First, we show that all strictly individually rational payoffs in $C$ can be supported by a 1 - memory SPE.

Proposition 4 For any payoff profile $u \in \mathcal{U}^{0} \cap C$ there exists a 1 - memory SPE strategy profile $f$ with $U^{\infty}(f)=u$.

When $n>2$, the proof of Proposition 4 for the case of a payoff profile $u \in \mathcal{U}^{0} \cap C$ involves constructing a confusion proof simple strategy profile $\left(\pi^{(0)}, \ldots, \pi^{(n)}\right)$ such that $u=V^{\infty}\left(\pi^{(0)}\right)$ and for each player $i \in N$ the punishment path $\pi^{(i)}$ involve playing first a finite sequence of action profiles with a payoff approximately close the minmax payoff $v_{i}$ for player $i$ and then playing the equilibrium path $\pi^{(0)}$. In the case of two players, the punishment phase for the two players are identical and consists of a finite sequence that involve plays that induce payoffs close to the mutual minmax payoffs followed by playing the equilibrium path $\pi^{(0)}$.

Since any strictly individually rational payoff $u \in \mathcal{U}$ can be approximated by payoff profiles in the set $\mathcal{U}^{0}$, we shall next show, using the previous result, that any individually rational payoff $u \in \mathcal{U}$ can be approximately implemented by a 1 - memory SPE strategy profile. ${ }^{14}$

Theorem 3 Suppose that $\mathcal{U}^{0}$ is nonempty. Then, for all $u \in \mathcal{U}$ and $\zeta>0$ there exists a 1 memory SPE strategy profile $f$ with $\left\|U^{\infty}(f)-u\right\|<\zeta$.

\section{Time Dependent Strategies}

The notion of a 1 - memory strategy implies that any such strategy cannot depend on the calendar time. In particular, if $\pi$ is the outcome path that a 1 - memory strategy $f$ induces, then $\pi^{t+1}=\pi^{r+1}$ provided that $\pi^{t}=\pi^{r}$. Thus, either the action profile prescribed in some date never repeats itself, or it will form a loop. As we have mentioned before, these restrictions imply that, in general, not all payoff vectors can be supported by 1 - memory strategies.

\footnotetext{
${ }^{14}$ By a similar argument as that in the proof of Proposition 4, it can also be shown that any $u \in \operatorname{int}\left(\mathcal{U}^{0}\right) \cap V^{\infty}\left(\Pi^{c p}\right)$ can also be implemented exactly by a 1 - memory subgame perfect equilibrium profile. This can be established by first noting that for any such $u \in \operatorname{int}\left(\mathcal{U}^{0}\right) \cap V^{\infty}\left(\Pi^{c p}\right)$ there exists another payoff $u^{\prime} \in \mathcal{U}^{0} \cap C$ such that $u \geq u^{\prime}$.
} 
Important differences appear when time dependent 1 - memory strategies are allowed. Formally, a strategy $f_{i} \in F_{i}$ for player $i$ is a time dependent 1 - memory strategy if $f_{i}(h)=f_{i}(\bar{h})$ for all $h, \bar{h} \in H_{t}$ satisfying $T(h)=T(\bar{h})$ and all $t \in \mathbb{N}$. Thus, a time dependent 1 - memory strategy allows for $f_{i}(h)$ to differ from $f_{i}(\bar{h})$ even if $T(h)=T(\bar{h})$, as long as $h$ and $\bar{h}$ have different lengths. Thus, time dependence implies that all outcome paths (and thus all payoff vectors) can be sustained by time dependent 1 - memory strategy profiles.

We are interested in supporting SPE payoffs with time dependent 1 - memory SPE strategies. Regarding this goal, similar considerations apply as in Sections 5 with the additional property that with time dependence we can strengthen our approximate implementation results to exact implementation. For example, the following is the analogue of Theorem 1 for time dependent 1 memory strategies that can be obtained by a similar proof.

Theorem 4 Let $\varepsilon \geq 0$ and $u$ be an $\varepsilon$ - strictly enforceable SPE payoff described by the simple strategy $\left(\hat{\pi}^{(0)}, \ldots, \hat{\pi}^{(n)}\right)$. Then, there exists a time dependent $1-$ memory $\varepsilon-C P E f$ with $U(f)=u$, provided that either $n \geq 3$, or $n=2$ and $\hat{\pi}^{(1)}=\hat{\pi}^{(2)}$.

From the above it should be clear that the advantage of using time dependent strategies is that payoffs can be supported exactly, and not only approximately. The same applies in the context of our Folk Theorems. In fact, there is no longer the need to focus on confusion-proof payoffs, since any payoff can be supported by a time dependent 1 - memory strategy.

Formally, for all $\delta \in(0,1)$ and $\alpha \in \mathcal{U}^{0}$ let $\tilde{\Lambda}(\alpha, \delta) \subset \Pi$ be the set of all outcome paths $\pi \in \Pi$ such that $V_{i}^{t}(\pi) \geq \alpha_{i}$ for all $i \in N$ and $t \in \mathbb{N}$. Also, denote the set of payoff vectors that are supported by the set $\tilde{\Lambda}(\alpha, \delta)$ by

$$
\tilde{C}(\alpha, \delta)=\left\{u \in \mathbb{R}^{n}: u=V(\pi, \delta) \text { for some } \pi \in \tilde{\Lambda}(\alpha, \delta)\right\}
$$

Then, because any payoff can be supported by a time dependent 1 - memory strategy, the following analogue of Proposition 3 can be obtained by a similar proof.

Proposition 5 Suppose that either $\operatorname{dim}(\mathcal{U})=n$ or $n=2$ and $\mathcal{U}^{0} \neq \emptyset$. Then for all $\alpha \in \mathcal{U}^{0}$, there exists $\bar{\delta} \in(0,1)$ such that for all $\delta \geq \bar{\delta}$ the following holds: for all payoffs $u \in \tilde{C}(\alpha, \delta)$ there exists time dependent 1 - memory SPE strategy $f$ with $U(f, \delta)=u$. 
Consequently, with the use of time dependent strategies the 1 - memory Folk Theorem can be stated without any approximations.

Theorem 5 For all payoffs $u \in \operatorname{int}\left(\mathcal{U}^{0}\right)$, there exists $\bar{\delta} \in(0,1)$ such that for all $\delta \geq \bar{\delta}$ there exists time dependent 1 - memory SPE strategy $f$ with $U(f, \delta)=u$.

\section{A Appendix: Robustness of the 2 - player Example}

\section{A.1 Perturbing the stage game payoffs and the discount factor}

We consider a perturbed version of the original game, where $\epsilon_{1}, \epsilon_{2}, \rho_{1}, \rho_{2}$ are sufficiently small and possibly negative:

\begin{tabular}{|c|c|c|}
\hline $1 \backslash 2$ & $a$ & $b$ \\
\hline$a$ & $\left(4+\epsilon_{1}, 4+\epsilon_{2}\right)$ & $\left(2+\rho_{1}, 5\right)$ \\
\hline$b$ & $\left(5,2+\rho_{2}\right)$ & $(0,0)$ \\
\hline
\end{tabular}

We will prove that there is an open neighborhood of values for $\epsilon_{1}, \epsilon_{2}, \rho_{1}, \rho_{2}$ and $\delta$, for which the (full memory) SPE payoff of $\left(4+\epsilon_{1}, 4+\epsilon_{2}\right)$ cannot be obtained by any 1 - memory SPE strategies.

As before, $s_{i} \in S_{i}=[0,1]$ refers to the action (the probability assigned to $a$ ) by player $i$ in the stage game. Note, also that for sufficiently small values of $\epsilon_{1}, \epsilon_{2}, \rho_{1}, \rho_{2}$, the minmax payoff is $\left(2+\rho_{i}\right)$ for player $i, m^{1}=(a, b), m^{2}=(b, a)$ and both $m^{1}$ and $m^{2}$ are Nash equilibria. The mutual minmax profile is $\bar{m}=\left(m_{1}^{2}, m_{2}^{1}\right)=(b, b)$.

Now suppose the above game is played infinitely often. If there are no restrictions on the memory then the payoff of $\left(4+\epsilon_{1}, 4+\epsilon_{2}\right)$ is SPE: play $a$ at each date with the threat of playing $m^{i}$ forever if $i$ deviates from $(a, a), i=1,2$ (further deviations are ignored). This strategy profile defined above is subgame perfect, provided that $\delta$ satisfies the following inequality

$$
\delta \geq \frac{1-\epsilon_{i}}{3-\rho_{i}} \text { for all } i=1,2
$$

To establish our claim suppose that, contrary to our claim, the payoff of $\left(4+\epsilon_{1}, 4+\epsilon_{2}\right)$ can be supported by a 1 - memory $\operatorname{SPE} f$. But then there exists functions $g_{i}:[0,1]^{2} \rightarrow[0,1]$ for all $i=1,2$ such that $f_{i}(h)=g_{i}(T(h))$ for all $h \in H \backslash H_{0}$. 
First, we will show that the payoff of $\left(4+\epsilon_{1}, 4+\epsilon_{2}\right)$ can only be obtained by repeating $(a, a)$ forever. Let $p_{1}=f_{1}\left(H_{0}\right), q_{1}=f_{2}\left(H_{0}\right), p_{t}=g_{1}\left(p_{t-1}, q_{t-1}\right)$ and $q_{t}=g_{2}\left(p_{t-1}, q_{t-1}\right)$ for all $t \in \mathbb{N}$. Since $U_{1}(f)=4+\epsilon_{1}$ and $U_{2}(f)=4+\epsilon_{2}$, it follows that

$$
\left.8+\sum_{i=1}^{2} \epsilon_{i}=\sum_{i=1}^{2} U_{i}(f)=(1-\delta) \sum_{t=1}^{\infty} \delta^{t-1}\left(\left(8+\epsilon_{1}+\epsilon_{2}\right) p_{t} q_{t}+\left(7+\rho_{1}\right)\left(p_{t}\left(1-q_{t}\right)\right)+\left(7+\rho_{2}\right) q_{t}\left(1-p_{t}\right)\right)\right) .
$$

Since $\left(\left(8+\epsilon_{1}+\epsilon_{2}\right) p_{t} q_{t}+\left(7+\rho_{1}\right)\left(p_{t}\left(1-q_{t}\right)\right)+\left(7+\rho_{2}\right) q_{t}\left(1-p_{t}\right)\right) \leq 8+\epsilon_{1}+\epsilon_{2}$ for small values of $\epsilon_{1}, \epsilon_{2}, \rho_{1}, \rho_{2}$, condition (11) holds only if $p_{t}=q_{t}=1$ for all $t \in \mathbb{N}$. Hence, it follows that $f_{i}\left(H_{0}\right)=g_{i}(1,1)=1$ for all $i=1,2$.

Next, for any $q \in S_{2}=[0,1]$ consider a deviation by player 2 to a strategy $\bar{f}_{2}$ defined by $\bar{f}_{2}\left(H_{0}\right)=q$ and $\bar{f}_{2}(h)=1$ for all $h \in H \backslash H_{0}$. Then,

$$
\begin{aligned}
U_{2}\left(f_{1}, \bar{f}_{2}\right) \geq & (1-\delta)\left[\left(4+\epsilon_{2}\right) q+5(1-q)\right]+(1-\delta) \delta\left[\left(4+\epsilon_{2}\right) g_{1}(1, q)\right. \\
& \left.\quad+\left(2+\rho_{2}\right)\left(1-g_{1}(1, q)\right)\right]+\left(2+\rho_{2}\right) \delta^{2} \\
= & (1-\delta)\left(5-q+\epsilon_{2} q\right)+\left(2+\rho_{2}\right) \delta+\delta(1-\delta)\left(2+\epsilon_{2}-\rho_{2}\right) g_{1}(1, q) .
\end{aligned}
$$

Since $f$ is a SPE, $4+\epsilon_{2}=U_{2}(f) \geq U_{2}\left(f_{1}, \bar{f}_{2}\right)$ implies

$$
g_{1}(1, q) \leq \frac{\left(1-\epsilon_{2}\right) q}{\left(2+\epsilon_{2}-\rho_{2}\right) \delta}+\frac{\left(3-\rho_{2}\right) \delta+\epsilon_{2}-1}{\left(2+\epsilon_{2}-\rho_{2}\right) \delta(1-\delta)}
$$

Symmetrically, for all $p \in S_{1}=[0,1]$

$$
g_{2}(p, 1) \leq \frac{\left(1-\epsilon_{1}\right) p}{\left(2+\epsilon_{1}-\rho_{1}\right) \delta}+\frac{\left(3-\rho_{1}\right) \delta+\epsilon_{1}-1}{\left(2+\epsilon_{1}-\rho_{1}\right) \delta(1-\delta)} .
$$

Consider next the strategy $\bar{f}_{1}$ for player 1 defined by $\bar{f}_{1}(h)=1$ for all $h \in H$. Note that for all $q \in S_{2}$

$$
\begin{aligned}
U_{1}\left(\bar{f}_{1}, f_{2} \mid(1, q)\right) & \geq(1-\delta)\left[\left(4+\epsilon_{1}\right) g_{2}(1, q)+\left(2+\rho_{1}\right)\left(1-g_{2}(1, q)\right)\right]+\left(2+\rho_{1}\right) \delta \\
& =\left(2+\rho_{1}\right)+(1-\delta)\left(2+\epsilon_{1}-\rho_{1}\right) g_{2}(1, q)
\end{aligned}
$$

and

$$
\begin{aligned}
U_{1}(f \mid(1, q)) \leq & (1-\delta)\left[\left(4+\epsilon_{1}\right) g_{1}(1, q) g_{2}(1, q)+\left(2+\rho_{1}\right) g_{1}(1, q)\left(1-g_{2}(1, q)\right)\right. \\
& \left.+5\left(1-g_{1}(1, q)\right) g_{2}(1, q)\right]+5 \delta \\
= & (1-\delta)\left[\left(2+\rho_{1}\right) g_{1}(1, q)+5 g_{2}(1, q)-\left(3-\epsilon_{1}+\rho_{1}\right) g_{1}(1, q) g_{2}(1, q)\right]+5 \delta \\
\leq & (1-\delta)\left[\left(2+\rho_{1}\right) g_{1}(1, q)+5 g_{2}(1, q)\right]+5 \delta .
\end{aligned}
$$


Again, since $f$ is SPE we have $U_{1}(f \mid(1, q)) \geq U_{1}\left(\bar{f}_{1}, f_{2} \mid(1, q)\right)$. This implies that

$$
g_{2}(1, q) \geq-\frac{\left(2+\rho_{1}\right) g_{1}(1, q)}{\left(3-\epsilon_{1}+\rho_{1}\right)}+\frac{2+\rho_{1}-5 \delta}{\left(3-\epsilon_{1}+\rho_{1}\right)(1-\delta)} \text { for all } q \in S_{2} .
$$

Finally, we use inequalities (12), (13) and (14) to show that player 1 has a profitable deviation from $f$, which contradicts the fact that $f$ is a SPE. To show this, consider player 1 deviating from the equilibrium path by choosing strategy $\tilde{f}_{1}$ defined by $\tilde{f}_{1}\left(H_{0}\right)=0$ and $\tilde{f}_{1}(h)=1$ for all $h \in H \backslash H_{0}$. Then,

$$
\begin{aligned}
U_{1}\left(\tilde{f}_{1}, f_{2}\right) & \geq 5(1-\delta)+\delta(1-\delta) u_{1}\left(1, g_{2}(0,1)\right)+\delta^{2}(1-\delta) u_{1}\left(1, g_{2}\left(1, g_{2}(0,1)\right)\right)+\left(2+\rho_{1}\right) \delta^{3} \\
& \geq 5(1-\delta)+\left(2+\rho_{1}\right) \delta+\delta^{2}(1-\delta)\left(2+\epsilon_{1}-\rho_{1}\right) g_{2}\left(1, g_{2}(0,1)\right) .
\end{aligned}
$$

The second inequality in the above follows from $u_{1}\left(1, g_{2}(0,1)\right) \geq 2+\rho_{1}$ and $u_{1}\left(1, g_{2}\left(1, g_{2}(0,1)\right)\right)=$ $\left(2+\epsilon_{1}-\rho_{1}\right) g_{2}\left(1, g_{2}(0,1)\right)+\left(2+\rho_{1}\right)$.

Next, we seek a lower bound on $g_{2}\left(1, g_{2}(0,1)\right)$. Note first that, by $(13)$, we have

$$
g_{2}(0,1) \leq \frac{\left(3-\rho_{1}\right) \delta+\epsilon_{1}-1}{\left(2+\epsilon_{1}-\rho_{1}\right) \delta(1-\delta)}:=\bar{g}_{2}(0,1) .
$$

This, together with (12), implies that

$$
\begin{aligned}
g_{1}\left(1, g_{2}(0,1)\right) & \leq \frac{\left(1-\epsilon_{2}\right) g_{2}(0,1)}{\left(2+\epsilon_{2}-\rho_{2}\right) \delta}+\frac{\left(3-\rho_{2}\right) \delta+\epsilon_{2}-1}{\left(2+\epsilon_{2}-\rho_{2}\right) \delta(1-\delta)} \\
& \leq \frac{\left(1-\epsilon_{2}\right) \bar{g}_{2}(0,1)}{\left(2+\epsilon_{2}-\rho_{2}\right) \delta}+\frac{\left(3-\rho_{2}\right) \delta+\epsilon_{2}-1}{\left(2+\epsilon_{2}-\rho_{2}\right) \delta(1-\delta)}:=\bar{g}_{1}\left(1, \bar{g}_{2}(0,1)\right) .
\end{aligned}
$$

But then, by (14), we obtain the desired lower bound on $g_{2}\left(1, g_{2}(0,1)\right)$ as follows:

$$
\begin{aligned}
g_{2}\left(1, g_{2}(0,1)\right) \geq & -\frac{\left(2+\rho_{1}\right) g_{1}\left(1, g_{2}(0,1)\right)}{\left(3-\epsilon_{1}+\rho_{1}\right)}+\frac{2+\rho_{1}-5 \delta}{\left(3-\epsilon_{1}+\rho_{1}\right)(1-\delta)} \geq \\
& -\frac{\left(2+\rho_{1}\right) \bar{g}_{1}\left(1, \bar{g}_{2}(0,1)\right)}{\left(3-\epsilon_{1}+\rho_{1}\right)}+\frac{2+\rho_{1}-5 \delta}{\left(3-\epsilon_{1}+\rho_{1}\right)(1-\delta)}:=\bar{g}_{2}\left(1, \bar{g}_{2}(0,1)\right) .
\end{aligned}
$$

Using the lower bound $\bar{g}_{2}\left(1, \bar{g}_{2}(0,1)\right)$ for $g_{2}\left(1, g_{2}(0,1)\right)$, we obtain

$$
U_{1}\left(\tilde{f}_{1}, f_{2}\right) \geq 5(1-\delta)+\left(2+\rho_{1}\right) \delta+\delta^{2}(1-\delta)\left(2+\epsilon_{1}-\rho_{1}\right) \bar{g}_{2}\left(1, \bar{g}_{2}(0,1)\right) .
$$

Now as $\delta \rightarrow 1 / 3, \epsilon_{j} \rightarrow 0$ and $\rho_{j} \rightarrow 0$ for all $j=1,2$, it follows that $\bar{g}_{2}(0,1) \rightarrow 0, \bar{g}_{1}\left(1, \bar{g}_{2}(0,1)\right) \rightarrow$ 0 and $\bar{g}_{2}\left(1, \bar{g}_{2}(0,1)\right) \rightarrow 1 / 6$, which imply that

$$
5(1-\delta)+\left(2+\rho_{1}\right) \delta+\delta^{2}(1-\delta)\left(2+\epsilon_{1}-\rho_{1}\right) \bar{g}_{2}\left(1, \bar{g}_{2}(0,1)\right) \rightarrow 4+\frac{2}{81} .
$$


Therefore, there exists $\phi^{*}>0, \epsilon_{j}^{*}>0$, and $\rho_{j}^{*}>0$ such that for all $|\delta-1 / 3|<\phi^{*},\left|\epsilon_{j}\right|<\epsilon_{j}^{*}$ and $\left|\rho_{j}\right|<\rho_{j}^{*}$ we have $U_{1}\left(\tilde{f}_{1}, f_{2}\right)>4+\epsilon_{1}$, delivering the required contradiction.

Thus, for all values of $\left(\delta, \epsilon_{1}, \epsilon_{2}, \rho_{1}, \rho_{2}\right)$ which reside in $B^{1} \cap B^{2}$ where $B^{1}=\left\{\left(\delta, \epsilon_{1}, \epsilon_{2}, \rho_{1}, \rho_{2}\right)\right.$ : $\left.4+\epsilon_{j}>5 \delta+(1-\delta)\left(2+\rho_{j}\right), j=1,2\right\}$ and $B^{2}=\left\{\left(\delta, \epsilon_{1}, \epsilon_{2}, \rho_{1}, \rho_{2}\right):|\delta-1 / 3|<\phi^{*},\left|\epsilon_{j}\right|<\epsilon_{j}^{*}\right.$ and $\left|\rho_{j}\right|<$ $\left.\rho_{j}^{*}, j=1,2\right\}$, there exists a SPE payoff which cannot be obtained by 1 - memory SPE.

\section{A.2 Contemporaneous $\varepsilon-$ equilibrium approximation}

Consider again the case of $\delta=1 / 3$ and $\epsilon_{1}=\epsilon_{2}=\rho_{1}=\rho_{2}=0$. We now show that exists $\eta>0$ and $\varepsilon^{\prime}>0$ such that no feasible payoff profile $u \in B_{\eta}(4,4)$ can be supported by a $1-$ memory $\varepsilon-\mathrm{CPE}$, for all $0 \leq \varepsilon \leq \varepsilon^{\prime}$.

To show this fix any $\eta>0$ and any feasible payoff profile $u \in B_{\eta}(4,4)$. We first show that if $\pi=\left\{\left(p_{t}, q_{t}\right)\right\}_{t=1}^{\infty}$ is such that $V(\pi)=u$, then $p_{1}$ and $q_{1}$ are both greater or equal to $1-3 \eta$. We shall demonstrate this for the case of $p_{1}$; the reasoning for the case of $q_{1}$ is analogous. Let $\gamma=1-3 \eta$ and $V_{t}=V_{1}^{t}(\pi)+V_{2}^{t}(\pi)$, for all $t \in \mathbb{N}$. Suppose, in order to reach a contradiction, that $p_{1}<\gamma$. Then

$$
V_{1}=\frac{2}{3}\left(8 p_{1} q_{1}+7 p_{1}\left(1-q_{1}\right)+7 q_{1}\left(1-p_{1}\right)\right)+\frac{V_{2}}{3}=\frac{2}{3}\left(7+p_{1} q_{1}-7\left(1-p_{1}\right)\left(1-q_{1}\right)\right)+\frac{V_{2}}{3}
$$

Note that $u \in B_{\eta}(4,4)$ implies that $V_{1}=u_{1}+u_{2}>8-2 \eta$. But then, by (15) and $p_{1}<\gamma$ we have

$$
8-2 \eta<\frac{2}{3}\left(7+\gamma q_{1}-7(1-\gamma)\left(1-q_{1}\right)\right)+\frac{V_{2}}{3}=\frac{2}{3}\left(-6 \gamma q_{1}+7\left(\gamma+q_{1}\right)\right)+\frac{V_{2}}{3} .
$$

But this implies that

$$
V_{2}>24-6 \eta+12 \gamma q_{1}-14\left(\gamma+q_{1}\right) \geq 10-6 \eta-2 \gamma=8
$$

Since $V_{2} \leq 8$, we have a contradiction.

Having shown that when $\eta>0$ is small $p_{1}$ and $q_{1}$ are near one, the rest of the proof is similar to that provided in the previous section. Therefore, as was done before, suppose contrary to our claim that, for some small $\eta>0$ and $\varepsilon \geq 0$, the payoff $u \in B_{\eta}(4,4)$ can be supported by a 1 - memory $\varepsilon$ - CPE $f$. Then, there exists functions $g_{i}:[0,1]^{2} \rightarrow[0,1]$ for all $i=1,2$ such that $f_{i}(h)=g_{i}(T(h))$ for all $h \in H \backslash H_{0}$. 
Now, for any $q \in S_{2}$ consider a deviation by player 2 to a strategy $\bar{f}_{2}$ defined by $\bar{f}_{2}\left(H_{0}\right)=q$ and $\bar{f}_{2}(h)=1$ for all $h \in H \backslash H_{0}$. Then,

$$
\begin{aligned}
U_{2}\left(f_{1}, \bar{f}_{2}\right) \geq & (1-\delta)\left[4 p_{1} q+5 p_{1}(1-q)+2\left(1-p_{1}\right) q\right] \\
& +(1-\delta) \delta\left[4 g_{1}\left(p_{1}, q\right)+2\left(1-g_{1}\left(p_{1}, q\right)\right)\right]+2 \delta^{2} \\
= & (1-\delta)\left(5 p_{1}-3 p_{1} q+2 q\right)+2 \delta+\delta(1-\delta) 2 g_{1}\left(p_{1}, q\right) .
\end{aligned}
$$

Since $f$ is $\varepsilon-\mathrm{CPE}, 4+\eta>U_{2}(f) \geq U_{2}\left(f_{1}, \bar{f}_{2}\right)-\varepsilon$ implies

$$
g_{1}\left(p_{1}, q\right)<\frac{4+\eta+\varepsilon-2 \delta}{2 \delta(1-\delta)}-\frac{5 p_{1}-3 p_{1} q+2 q}{2 \delta} .
$$

Symmetrically, for all $p \in S_{1}$

$$
g_{2}\left(p, q_{1}\right)<\frac{4+\eta+\varepsilon-2 \delta}{2 \delta(1-\delta)}-\frac{5 q_{1}-3 p q_{1}+2 p}{2 \delta} .
$$

Consider next the strategy $\bar{f}_{1}$ for player 1 defined by $\bar{f}_{1}(h)=1$ for all $h \in H$. Note that

$$
\begin{aligned}
U_{1}\left(\bar{f}_{1}, f_{2} \mid\left(p_{1}, q\right)\right) & \geq(1-\delta)\left[4 g_{2}\left(p_{1}, q\right)+2\left(1-g_{2}\left(p_{1}, q\right)\right)\right]+2 \delta \\
& =2+(1-\delta) 2 g_{2}\left(p_{1}, q\right)
\end{aligned}
$$

for all $q \in S_{2}$, and

$$
\begin{aligned}
U_{1}\left(f \mid\left(p_{1}, q\right)\right) \leq & (1-\delta)\left[4 g_{1}\left(p_{1}, q\right) g_{2}\left(p_{1}, q\right)+2 g_{1}\left(p_{1}, q\right)\left(1-g_{2}\left(p_{1}, q\right)\right)\right. \\
& \left.+5\left(1-g_{1}\left(p_{1}, q\right)\right) g_{2}\left(p_{1}, q\right)\right]+5 \delta \leq(1-\delta)\left(2 g_{1}\left(p_{1}, q\right)+5 g_{2}\left(p_{1}, q\right)\right)+5 \delta .
\end{aligned}
$$

Again, since $f$ is $\varepsilon-\mathrm{CPE}$ we have for all $q \in S_{2}$

$$
g_{2}\left(p_{1}, q\right) \geq-\frac{2 g_{1}\left(p_{1}, q\right)}{3}+\frac{2-5 \delta-\varepsilon}{3(1-\delta)} .
$$

Finally, we use inequalities (16), (17) and (18) to show that for small $\eta$ and $\varepsilon$ player 1 has a profitable deviation from $f$, which contradicts the fact that $f$ is $\varepsilon-\mathrm{CPE}$. To show this, consider player 1 deviating from the equilibrium path by choosing strategy $\tilde{f}_{1}$ defined by $\tilde{f}_{1}\left(H_{0}\right)=0, \tilde{f}_{1}\left(0, q_{1}\right)=p_{1}$, and $\tilde{f}_{1}(h)=1$ for all $h \in H \backslash\left(H_{0} \cup\left\{\left(0, q_{1}\right)\right\}\right)$. Then,

$$
\begin{aligned}
U_{1}\left(\tilde{f}_{1}, f_{2}\right) & \geq 5 q_{1}(1-\delta)+\delta(1-\delta) u_{1}\left(p_{1}, g_{2}\left(0, q_{1}\right)\right)+\delta^{2}(1-\delta) u_{1}\left(1, g_{2}\left(p_{1}, g_{2}\left(0, q_{1}\right)\right)\right)+2 \delta^{3} \\
& \geq 5 q_{1}(1-\delta)+2 \delta+\delta^{2}(1-\delta) 2 g_{2}\left(p_{1}, g_{2}\left(0, q_{1}\right)\right)-2 \delta(1-\delta)\left(1-p_{1}\right)
\end{aligned}
$$


because $u_{1}\left(p_{1}, g_{2}\left(0, q_{1}\right)\right) \geq 2 p_{1}$ and $u_{1}\left(1, g_{2}\left(p_{1}, g_{2}\left(0, q_{1}\right)\right)\right)=2 g_{2}\left(p_{1}, g_{2}\left(0, q_{1}\right)\right)+2$.

Next, we seek a lower bound on $g_{2}\left(p_{1}, g_{2}\left(0, q_{1}\right)\right)$. Note first that, by $(17)$, we have

$$
g_{2}\left(0, q_{1}\right) \leq \frac{4+\eta+\varepsilon-2 \delta}{2 \delta(1-\delta)}-\frac{5 q_{1}}{2 \delta}:=\bar{g}_{2}\left(0, q_{1}\right)
$$

This, together with (16), implies that

$$
\begin{aligned}
g_{1}\left(p_{1}, g_{2}\left(0, q_{1}\right)\right) & \leq \frac{4+\eta+\varepsilon-2 \delta}{2 \delta(1-\delta)}+\frac{\left(3 p_{1}-2\right) g_{2}\left(0, q_{1}\right)-5 p_{1}}{2 \delta} \\
& \leq \frac{4+\eta+\varepsilon-2 \delta}{2 \delta(1-\delta)}+\frac{\left(3 p_{1}-2\right) \bar{g}_{2}\left(0, q_{1}\right)-5 p_{1}}{2 \delta}:=\bar{g}_{1}\left(p_{1}, \bar{g}_{2}\left(0, q_{1}\right)\right),
\end{aligned}
$$

because for $\eta>0$ small enough, $\left(3 p_{1}-2\right)>0$ (due to $\left.p_{1} \geq 1-3 \eta\right)$. But then, by (18), we obtain the desired lower bound on $g_{2}\left(p_{1}, g_{2}\left(0, q_{1}\right)\right)$ as follows:

$$
\begin{aligned}
g_{2}\left(1, g_{2}(0,1)\right) & \geq-\frac{2 g_{1}\left(p_{1}, g_{2}\left(0, q_{1}\right)\right)}{3}+\frac{2-5 \delta-\varepsilon}{3(1-\delta)} \\
& \geq-\frac{2 \bar{g}_{1}\left(p_{1}, \bar{g}_{2}\left(0, q_{1}\right)\right)}{3}+\frac{2-5 \delta-\varepsilon}{3(1-\delta)}:=\bar{g}_{2}\left(p_{1}, \bar{g}_{2}\left(0, q_{1}\right)\right) .
\end{aligned}
$$

Using the lower bound $\bar{g}_{2}\left(p_{1}, \bar{g}_{2}\left(0, q_{1}\right)\right)$ for $g_{2}\left(p_{1}, g_{2}\left(0, q_{1}\right)\right)$, we obtain

$$
U_{1}\left(\tilde{f}_{1}, f_{2}\right) \geq 5 q_{1}(1-\delta)+2 \delta+\delta^{2}(1-\delta) 2 \bar{g}_{2}\left(p_{1}, \bar{g}_{2}\left(0, q_{1}\right)\right)-2 \delta(1-\delta)\left(1-p_{1}\right)
$$

Now set $\delta=1 / 3$. Then as $\eta \rightarrow 0$ and $\varepsilon \rightarrow 0$, we have $p_{1} \rightarrow 1, q_{1} \rightarrow 1, \bar{g}_{2}\left(0, q_{1}\right) \rightarrow 0$, $\bar{g}_{1}\left(p_{1}, \bar{g}_{2}\left(0, q_{1}\right)\right) \rightarrow 0, \bar{g}_{2}\left(p_{1}, \bar{g}_{2}\left(0, q_{1}\right)\right) \rightarrow 1 / 6$ and

$$
U_{1}\left(\tilde{f}_{1}, f_{2}\right) \rightarrow 4+\frac{2}{81}
$$

Since $U_{1}(f)<4+\eta$, this implies that for $\eta$ and $\varepsilon>0$ sufficiently small $U_{1}\left(\tilde{f}_{1}, f_{2}\right)>U_{1}(f)+\varepsilon$. But this is a contradiction.

\section{B Appendix: Proofs}

Proof of Remark 1. Suppose that $\left(\pi^{(0)}, \pi^{(1)}, \pi^{(2)}\right)$ is confusion proof. Consider the four possible values that $\Omega(\{1,1\},\{2,1\})$ can take. First, note that it cannot be that $\Omega(\{1,1\},\{2,1\})=\{1,2\}$; otherwise, this means that $\pi^{(1), 1} \neq \pi^{(2), 1}$, while by part 3 of Definition 2 we have $\pi^{(1)}=\pi^{(2)}$. 
Second, if $\Omega(\{1,1\},\{2,1\})=\emptyset$ then $\pi^{(1), 1}=\pi^{(2), 1}$. Proceeding by induction, assume that $\pi^{(1), r}=\pi^{(2), r}$ for all $r=1, \ldots, t-1$. Then, $\Omega(\{1, t-1\},\{2, t-1\})=\emptyset$ implies that $\pi^{(1), t}=\pi^{(2), t}$. Hence, $\pi^{(1)}=\pi^{(2)}$.

Suppose that $\Omega(\{1,1\},\{2,1\})=\{1\}$, which means that $\pi_{1}^{(1), 1} \neq \pi_{1}^{(2), 1}$. By part 2 of Definition 2 we have $\pi^{(1), 1}=\pi^{(1), 2}=\pi^{(2), 2}$. Proceeding by induction as above, one can prove that $\pi^{(1), t}=\pi^{(1), t+1}$ for all $t \in \mathbb{N}$ and that $\pi^{(1), t}=\pi^{(2), t}$ for all $t \geq 2$. This completes the proof, since the remaining case $(\Omega(\{1,1\},\{2,1\})=\{2\})$ is just analogous to this one.

Proof of Proposition 1. (Sufficiency) Let $\left(\pi^{(0)}, \ldots, \pi^{(n)}\right)$ be a confusion-proof profile of outcome paths. Let $i \in N$ and define $f_{i}$ as follows: for any $h \in H, j \in\{0, \ldots, n\}, l \in N$ and $t \in \mathbb{N}$

$$
f_{i}(h)= \begin{cases}\pi_{i}^{(j), t+1} & \text { if } T(h)=\pi^{(j), t} \\ \pi_{i}^{(l), 1} & \text { if } T(h)=\left(s_{l}, \pi_{-l}^{(j), t}\right) \text { and } s_{l} \neq \pi_{l}^{(j), t} \\ \pi_{i}^{(0), 1} & \text { otherwise. }\end{cases}
$$

Now we show that $f$ is a well defined function. First, suppose that $\pi^{(j), t}=\pi^{(k), r}$ for some $k, j \in\{0,1, \ldots, n\}$ and $r, t \in \mathbb{N}$. Then, $f$ is well defined if $\pi^{(k), r+1}=\pi^{(j), t+1}$. Since $\left(\pi^{(0)}, \ldots, \pi^{(n)}\right)$ is confusion-proof, it follows from part 1 of Definition 2 that this is indeed the case.

Second, suppose that $\pi^{(k), r}=\left(s_{l}, \pi_{-l}^{(j), t}\right)$ and $s_{l} \neq \pi_{l}^{(j), t}$ for $k, j \in\{0,1, \ldots, n\}, l \in N$ and $r, t \in \mathbb{N}$. Then, $f$ is well defined only if $\pi^{(k), r+1}=\pi^{(l), 1}$. Since $\left(\pi^{(0)}, \ldots, \pi^{(n)}\right)$ is confusion-proof and $\Omega(\{k, r\},\{j, t\})=\{l\}$, it follows from part 2 of Definition 2 that this is indeed the case.

Finally, suppose that $\left(s_{l}, \pi_{-l}^{(j), t}\right)=\left(s_{k}, \pi_{-k}^{(m), r}\right), s_{k} \neq \pi_{k}^{(m), r}$ and $s_{l} \neq \pi^{(j), t}$ for some $j, m \in$ $\{0,1, \ldots, n\}, k, l \in N$ and $r, t \in \mathbb{N}$. Then $f$ is well defined only if $\pi^{(l), 1}=\pi^{(k), 1}$. Note that it must be that $s_{l}=\pi_{l}^{(m), r}$ and $s_{k}=\pi_{k}^{(j), t}$. Hence, $\pi_{l}^{(m), r} \neq \pi_{l}^{(j), t}$ and $\pi_{k}^{(m), r} \neq \pi_{k}^{(j), t}$, implying that $\Omega(\{m, r\},\{j, t\})=\{k, l\}$. Since $\left(\pi^{(0)}, \ldots, \pi^{(n)}\right)$ is confusion-proof, it follows from part 3 of Definition 2 that $\pi^{(l), 1}=\pi^{(k), 1}$.

It is clear that the strategy $f=\left(f_{1} \ldots, f_{n}\right)$ has 1 - memory, since, by definition, $f_{i}$ depends only on $T(h)$ for all $i \in N$.

Note, also that $f$ has the following property: $\pi(f)=\pi^{(0)}$ and if player $i \in N$ deviates unilaterally in phase $t$ in any state $j$, then $\pi^{(i)}$ will be played starting from period $t+1$. Therefore, $f$ defined by $\left(\pi^{(0)}, \ldots, \pi^{(n)}\right)$ is a 1 - memory simple strategy.

(Necessity) Let $f$ be a 1 - memory simple strategy represented by $\left(\pi^{(0)}, \ldots, \pi^{(n)}\right)$. Let $i, j \in$ $\{0, \ldots, n\}$ and $t, r \in \mathbb{N}$. 
Suppose that $\Omega(\{i, t\},\{j, r\})=\emptyset$. Then, $\pi^{(i), t}=\pi^{(j), r}$. Let $h_{1}=\left(\pi^{(i), t}\right)$ and $h_{2}=\left(\pi^{(j), r}\right)$. Since $T\left(h_{1}\right)=h_{1}=h_{2}=T\left(h_{2}\right)$ and $f$ has 1 - memory, we have $f\left(h_{1}\right)=f\left(h_{2}\right)$. But then part 1 of Definition 2 is satisfied because $f\left(h_{1}\right)=\pi^{(i), t+1}$ and $f\left(h_{2}\right)=\pi^{(j), r+1}$.

Suppose next that $\Omega(\{i, t\},\{j, r\})=\{k\}$ for some $k \in N$. Then, $\pi_{l}^{(i), t}=\pi_{l}^{(j), r}$ for all $l \neq k$, while $\pi_{k}^{(i), t} \neq \pi_{k}^{(j), r}$. Consider $s_{k}=\pi_{k}^{(i), t}$ and $\bar{s}_{k}=\pi_{k}^{(j), r}$. Then, $\left(s_{k}, \pi_{-k}^{(j), r}\right)=\pi^{(i), t}$ and since $f$ is a 1 - memory simple strategy, it follows that

$$
\pi^{(k), 1}=f\left(\left(s_{k}, \pi_{-k}^{(j), r}\right)\right)=f\left(\pi^{(i), t}\right)=\pi^{(i), t+1} .
$$

Similarly, $\left(\bar{s}_{k}, \pi_{-k}^{(i), t}\right)=\pi^{(j), r}$ and so,

$$
\pi^{(k), 1}=f\left(\left(\bar{s}_{k}, \pi_{-k}^{(i), t}\right)\right)=f\left(\pi^{(j), r}\right)=\pi^{(j), r+1} .
$$

Hence, $\pi^{(k), 1}=\pi^{(j), r+1}=\pi^{(i), t+1}$ and part 2 of Definition 2 is satisfied.

Finally, suppose that $\Omega(\{i, t\},\{j, r\})=\{k, l\}$ for some $k, l \in N$. Then, $\pi_{m}^{(i), t}=\pi_{m}^{(j), r}$ for all $m \notin\{k, l\}$, while $\pi_{k}^{(i), t} \neq \pi_{k}^{(j), r}$ and $\pi_{l}^{(i), t} \neq \pi_{l}^{(j), r}$. Consider $s_{k}=\pi_{k}^{(j), r}$ and $s_{l}=\pi_{l}^{(i), t}$. Then, $\left(s_{l}, \pi_{-l}^{(j), r}\right)=\left(s_{k}, \pi_{-k}^{(i), t}\right)$ and since $f$ is a 1 - memory simple strategy, it follows that

$$
\pi^{(l), 1}=f\left(\left(s_{l}, \pi_{-l}^{(j), r}\right)\right)=f\left(\left(s_{k}, \pi_{-k}^{(i), t}\right)\right)=\pi^{(k), 1} .
$$

Hence, by induction, $\pi^{(l)}=\pi^{(k)}$ and part 3 of Definition 2 is satisfied.

Proof of Theorem 1. Let $\varepsilon \geq 0, \eta>0$ and $u$ be an $\varepsilon$ - strictly enforceable SPE payoff vector decribed by $\left(\hat{\pi}^{(0)}, \ldots, \hat{\pi}^{(n)}\right)$. For all $j \in\{0,1, \ldots, n\}$ and $i \in N$, define $\zeta_{i}^{(j)}$ by

$$
\zeta_{i}^{(j)}=\inf _{t \in \mathbb{N}}\left(V_{i}^{t}\left(\hat{\pi}^{(j)}\right)-\left((1-\delta) \max _{s_{i}} u_{i}\left(s_{i}, \hat{\pi}_{-i}^{(j), t}\right)+\delta V_{i}\left(\hat{\pi}^{(i)}\right)\right)\right) .
$$

Let $\gamma$ be defined by

$$
\gamma=\min \left\{\eta, \frac{1}{2}\left(\min _{j \in\{0,1, \ldots, n\}, i \in N}\left\{\zeta_{i}^{(j)}\right\}+\varepsilon\right)\right\} .
$$

It follows that $\gamma>0$ since $\eta>0$ and $u$ is an $\varepsilon$ - strictly enforceable SPE payoff vector.

Let $\psi>0$ be such that $d(x, y)<\psi$ implies $\left|u_{i}(x)-u_{i}(y)\right|<\gamma$ and $\mid \max _{z_{i}} u_{i}\left(z_{i}, x_{-i}\right)-$ $\max _{z_{i}} u_{i}\left(z_{i}, y_{-i}\right) \mid<\gamma$, for all $i \in N$. Since $S_{i}$ is connected for all $i \in N$ it follows that for every $j=0,1, \ldots n$ and $t \in \mathbb{N}, B_{\psi}\left(\hat{\pi}^{(j), t}\right) \cap S$ is uncountable. Thus, we can construct a simple outcome paths $\left(\bar{\pi}^{(0)}, \bar{\pi}^{(1)}, \ldots, \bar{\pi}^{(n)}\right)$ satisfying the conditions described in Lemma 1 . Thus, $\left(\bar{\pi}^{(0)}, \bar{\pi}^{(1)}, \ldots, \bar{\pi}^{(n)}\right)$ 
is confusion proof. Therefore, by Proposition 1, there exists a 1 - memory strategy profile $f$ that is represented by it. Moreover, $\gamma \leq \eta$ implies $\left|U_{i}(f)-u_{i}\right|=\left|V_{i}\left(\bar{\pi}^{(0)}\right)-V_{i}\left(\hat{\pi}^{(0)}\right)\right|<\eta$ for all $i$.

To complete the proof we need to show $f$ is $\varepsilon-\mathrm{CPE}$. Fix any $t \in \mathbb{N}, i \in N$ and $j \in\{0,1, \ldots, n\}$. Since $V_{i}^{t}\left(\hat{\pi}^{(j)}\right)-\gamma<V_{i}^{t}\left(\bar{\pi}^{(j)}\right), V_{i}\left(\bar{\pi}^{(i)}\right)<V_{i}\left(\hat{\pi}^{(i)}\right)+\gamma$ and $\max _{s_{i}} u_{i}\left(s_{i}, \bar{\pi}_{-i}^{(j), t}\right)<\max _{s_{i}} u_{i}\left(s_{i}, \hat{\pi}_{-i}^{(j), t}\right)+\gamma$, it follows from (19) that

$$
\begin{aligned}
& V_{i}^{t}\left(\bar{\pi}^{(j)}\right)-(1-\delta) \max _{s_{i}} u_{i}\left(s_{i}, \bar{\pi}_{-i}^{(j), t}\right)-\delta V_{i}\left(\bar{\pi}^{(i)}\right)> \\
& \quad V_{i}^{t}\left(\hat{\pi}^{(j)}\right)-(1-\delta) \max _{s_{i}} u_{i}\left(s_{i}, \hat{\pi}_{-i}^{(j), t}\right)-\delta V_{i}\left(\hat{\pi}^{(i)}\right)-2 \gamma \geq \zeta_{i}^{(j)}-2 \gamma \geq 2 \gamma-\varepsilon-2 \gamma=-\varepsilon .
\end{aligned}
$$

Hence, it does not pay player $i$ to deviate from the path induced by state $(j)$ by more than $\varepsilon$. Thus, $f$ is a confusion-proof $\varepsilon-\mathrm{CPE}$.

Proof of Proposition 3. We consider the two cases of $n \geq 3$ (Case A) and the $n=2$ (Case B) separately.

Case A: $n \geq 3$ and $\operatorname{dim}(\mathcal{U})=n$.

For convenience, in this case we normalize payoffs so that $v_{i}=0$ for all $i \in N$.

Fix any $\alpha \in \mathcal{U}^{0}$. Then, by Theorem 1 (Step 1) in Abreu, Dutta, and Smith (1994), for all $i \in N$ there exists $y^{i} \in \mathcal{U}^{0}$ satisfying the following property: for all $i, j \in N$ with $j \neq i, y_{i}^{i}<\alpha_{i}$ and $y_{i}^{i}<y_{i}^{j}$.

Define $\gamma_{i}^{\prime}>0$ to be such that $2 \gamma_{i}^{\prime}=\min \left\{\alpha_{i},\left\{y_{i}^{j}\right\}_{j \neq i}\right\}-y_{i}^{i}$. Let $\alpha_{i}^{\prime}=y_{i}^{i}+\gamma_{i}^{\prime}$ for each $i$. Clearly, $\gamma_{i}^{\prime}<\alpha_{i}^{\prime}<\alpha_{i}$. Also, by the properties of $\left(y^{1}, \ldots, y^{n}\right)$ we have $\gamma_{i}^{\prime}>0$ for all $i$.

Next, let $\gamma=\min _{i} \min \left\{\gamma_{i}^{\prime}, y_{i}^{i}\right\}$ and $M=\max _{i} \max _{s \in S}\left|u_{i}(s)\right|$. Also, suppose $T \in \mathbb{N}$ is such that

$$
T \geq \frac{4 M}{\gamma}=\frac{M}{\gamma / 4}
$$

Denote $D_{k}$ to be the set of achievable payoff in the finite game that consists of repeating the one-shot game $k$ times, and in which payoffs consist of the average of the payoffs obtained in the $k$ stages. Also, let $K \in \mathbb{N}$ be such that $D \subseteq \cup_{x \in D_{K}} B_{\gamma / 2}(x)$ (see Sorin (1992, Proposition 1.3.)). Finally, denote $\bar{\delta} \in(0,1)$ to be such that $\delta \geq \bar{\delta}$ implies

$$
\begin{aligned}
& \frac{1-\delta^{T+1}}{1-\delta}>T \\
& \frac{\delta^{T}}{1-\delta^{T}}>\frac{8 M}{\gamma}=\frac{2 M}{\gamma / 4} \text { and } \\
& \sup _{x \in[-M-\gamma, M+\gamma]^{K}}\left|\frac{1-\delta}{1-\delta^{K}} \sum_{k=1}^{K} \delta^{k-1} x_{k}-\frac{1}{K} \sum_{k=1}^{K} x_{k}\right|<\frac{\gamma}{4} .
\end{aligned}
$$


Now fix any $\delta \geq \bar{\delta}$ and consider any $u \in C(\alpha, \delta)$. We will show that there is a $1-$ memory SPE strategy profile $f$ with $U(f)=u$.

Let $\pi \in \Lambda(\alpha, \delta)$ be such that it satisfies $V_{i}(\pi, \delta)=u_{i}$ for all $i \in N$. Since $\pi$ is a confusion-proof single path, by Proposition 2, to complete the proof and show that $u$ can be sustained by a 1 - memory SPE it is sufficient to establish that there exists a strictly enforceable simple strategy profile given by $\left(\pi^{(0)}, \pi^{(1)}, \ldots, \pi^{(n)}\right)$ such that $\pi^{(0)}=\pi$.

We first start by constructing for each player $i$ a punishment path $\pi^{(i)}$. This path consists of playing $m^{i}$ for the first $T$ periods followed by a path $\widehat{\pi}^{(i)}$ yielding a payoff to player $i$ that is less than $\alpha_{i}^{\prime}$ and bounded away from zero (the minmax payoff) by $\gamma / 4$. Furthermore, we need $V_{i}^{t}\left(\widehat{\pi}^{(i)}\right) \geq V_{i}\left(\widehat{\pi}^{(i)}\right)$ for all $i \in N$ and $t \in \mathbb{N}$ to prevent player $i$ to deviate at latter stages of his punishment path.

To construct such a path, let $x^{i} \in D_{K}$ be such that $\left\|x^{i}-y^{i}\right\|<\gamma / 2$ for all $i \in N$. Then, by the definitions of $y^{i}, \alpha_{i}^{\prime}, \gamma_{i}^{\prime}$ and $\gamma$, the following hold for all $i$ :

$$
\begin{aligned}
& x_{i}^{i}<y_{i}^{i}+\frac{\gamma}{2}=\alpha_{i}^{\prime}-\gamma_{i}^{\prime}+\frac{\gamma}{2} \leq \alpha_{i}^{\prime}-\frac{\gamma}{2} \\
& x_{i}^{i}>y_{i}^{i}-\frac{\gamma}{2} \geq \gamma-\frac{\gamma}{2}=\frac{\gamma}{2} \\
& x_{i}^{j}>y_{i}^{j}-\frac{\gamma}{2} \geq y_{i}^{i}+2 \gamma_{i}^{\prime}-\frac{\gamma}{2}=\alpha_{i}^{\prime}+\gamma_{i}^{\prime}-\frac{\gamma}{2} \geq \alpha_{i}^{\prime}+\frac{\gamma}{2} .
\end{aligned}
$$

Now, let $\left\{s^{i, k}\right\}_{k=1}^{K}$ be such that

$$
\frac{1}{K} \sum_{k=1}^{K} u_{j}\left(s^{i, k}\right)=x_{j}^{i},
$$

for all $i, j \in N$. Next, consider any $t^{*}$ such that

$$
t^{*} \in \arg \min _{1 \leq t \leq K} \frac{1-\delta}{1-\delta^{K}}\left[\sum_{k=t}^{K} \delta^{k-t} u_{i}\left(s^{i, k}\right)+\sum_{k=1}^{t-1} \delta^{k+T-t} u_{i}\left(s^{i, k}\right)\right],
$$

Let $\widehat{\pi}^{(i)}$ consist of repetitions of $\left(s^{i, t^{*}}, s^{i, t^{*}+1}, \ldots, s^{i, K}, s^{i, 1}, \ldots, s^{i, t^{*}-1}\right)$. Then

$$
V_{i}^{t}\left(\widehat{\pi}^{(i)}\right) \geq V_{i}\left(\widehat{\pi}^{(i)}\right) \text { for all } i \text { and } t
$$

Also, using (23), it follows, respectively, from (24), (25) and (26) that for all $i$

$$
\begin{aligned}
& V_{i}\left(\widehat{\pi}^{(i)}\right)<x_{i}^{i}+\frac{\gamma}{4}<\alpha_{i}^{\prime}-\frac{\gamma}{2}+\frac{\gamma}{4}<\alpha_{i}^{\prime}, \\
& V_{i}\left(\widehat{\pi}^{(i)}\right)>x_{i}^{i}-\frac{\gamma}{4}>\frac{\gamma}{2}-\frac{\gamma}{4}=\frac{\gamma}{4} \text { and } \\
& V_{i}^{t}\left(\widehat{\pi}^{(j)}\right)>x_{i}^{j}-\frac{\gamma}{4}>\alpha_{i}^{\prime}+\frac{\gamma}{2}-\frac{\gamma}{4}=\alpha_{i}^{\prime}+\frac{\gamma}{4}>V_{i}\left(\widehat{\pi}^{(i)}\right) \text { for all } t \text { and } j \neq i .
\end{aligned}
$$


Finally, let $\pi^{(0)}=\pi$ and define the path $\pi^{(i)}$ by

$$
\pi^{(i), t}= \begin{cases}m^{i} & \text { if } t \leq T \\ \widehat{\pi}^{(i), t-T} & \text { if } t>T .\end{cases}
$$

We now show that the simple strategy defined by $\left(\pi^{(0)}, \ldots, \pi^{(n)}\right)$ supports $u$ as a strictly enforceable SPE payoff.

Let $i \in N$ be given. First, consider player $i$ deviating from the equilibrium path $\pi^{(0)}=\pi$ in period $t$. Then we have

$$
\begin{aligned}
& V_{i}^{t}(\pi)-\left((1-\delta) \max _{s_{i}} u_{i}\left(s_{i}, \pi_{-i}^{t}\right)+\delta V_{i}\left(\pi^{(i)}\right)\right)=V_{i}^{t}(\pi)-\left((1-\delta) \max _{s_{i}} u_{i}\left(s_{i}, \pi_{-i}^{t}\right)+\delta^{T+1} V_{i}\left(\widehat{\pi}^{(i)}\right)\right) \\
& \quad>\alpha_{i}^{\prime}-\left((1-\delta) M+\delta^{T+1} \alpha_{i}^{\prime}\right) .
\end{aligned}
$$

(The equality in the above follows from $V_{i}\left(\pi^{(i)}\right)=\delta^{T} V_{i}\left(\widehat{\pi}^{(i)}\right)$ and the inequality from $V_{i}^{t}(\pi) \geq \alpha_{i}>\alpha_{i}^{\prime}$ and from (28).) But then since $\alpha_{i}^{\prime}>(1-\delta) M+\delta^{T+1} \alpha_{i}^{\prime}$ is equivalent to

$$
\frac{1-\delta^{T+1}}{1-\delta}>\frac{M}{\alpha_{i}^{\prime}}
$$

and, by (21), (20) and the definition of $\gamma$,

$$
\frac{1-\delta^{T+1}}{1-\delta}>T \geq \frac{M}{\gamma / 4} \geq \frac{M}{\alpha_{i}^{\prime}}
$$

it follows that

$$
\inf _{t}\left[V_{i}^{t}(\pi)-\left((1-\delta) \max _{s_{i}} u_{i}\left(s_{i}, \pi_{-i}^{(0), t}\right)+\delta V_{i}\left(\pi^{(i)}\right)\right)\right]>0 .
$$

Second, consider a deviation from $\pi^{(i), t}$. If $t \leq T$ we have that

$$
V_{i}^{t}\left(\pi^{(i)}\right)-\left((1-\delta) \max _{s_{i}} u_{i}\left(s_{i}, m_{-i}^{i}\right)+\delta V_{i}\left(\pi^{(i)}\right)\right)=\delta^{T+1-t} V_{i}\left(\widehat{\pi}^{(i)}\right)-\delta^{T+1} V_{i}\left(\widehat{\pi}^{(i)}\right)>0 .
$$

(This follows from $V_{i}\left(\pi^{(i)}\right)=\delta^{T} V_{i}\left(\widehat{\pi}^{(i)}\right), \max _{s_{i}} u_{i}\left(s_{i}, m_{-i}^{i}\right)=0$ and $V_{i}\left(\widehat{\pi}^{(i)}\right)>0$.) If $t>T$, then

$$
\begin{aligned}
& V_{i}^{t}\left(\pi^{(i)}\right)-\left((1-\delta) \max _{s_{i}} u_{i}\left(s_{i}, \pi_{-i}^{(i), t}\right)+\delta V_{i}\left(\pi^{(i)}\right)\right) \\
& =V_{i}^{t-T}\left(\widehat{\pi}^{(i)}\right)-\left((1-\delta) \max _{s_{i}} u_{i}\left(s_{i}, \pi_{-i}^{(i), t}\right)+\delta^{T+1} V_{i}\left(\widehat{\pi}^{(i)}\right)\right) \\
& \geq V_{i}\left(\widehat{\pi}^{(i)}\right)-\left((1-\delta) M+\delta^{T+1} V_{i}\left(\widehat{\pi}^{(i)}\right)\right) .
\end{aligned}
$$

(The inequality in the above follow from (27).) Since $V_{i}\left(\widehat{\pi}^{(i)}\right)>(1-\delta) M+\delta^{T+1} V_{i}\left(\widehat{\pi}^{(i)}\right)$ is equivalent to

$$
\frac{1-\delta^{T+1}}{1-\delta}>\frac{M}{V_{i}\left(\widehat{\pi}^{(i)}\right)}
$$


and, by (20), (21) and (29),

$$
\frac{1-\delta^{T+1}}{1-\delta}>T \geq \frac{M}{\gamma / 4}>\frac{M}{V_{i}\left(\widehat{\pi}^{(i)}\right)}
$$

it follows that

$$
\inf _{t}\left[V_{i}^{t}\left(\pi^{(i)}\right)-\left((1-\delta) \max _{s_{i}} u_{i}\left(s_{i}, \pi_{-i}^{(i), t}\right)+\delta V_{i}\left(\pi^{(i)}\right)\right)\right]>0 .
$$

Finally, consider a deviation from $\pi^{(j), t}, j \neq i$. If $t>T$, then it follows from (30) that

$$
\begin{aligned}
& V_{i}^{t}\left(\pi^{(j)}\right)-\left((1-\delta) \max _{s_{i}} u_{i}\left(s_{i}, m_{-i}^{j}\right)+\delta V_{i}\left(\pi^{(i)}\right)\right) \\
& =V_{i}^{t-T}\left(\widehat{\pi}^{(j)}\right)-\left((1-\delta) \max _{s_{i}} u_{i}\left(s_{i}, m_{-i}^{j}\right)+\delta^{T+1} V_{i}\left(\widehat{\pi}^{(i)}\right)\right) \\
& >V_{i}\left(\widehat{\pi}^{(i)}\right)-\left((1-\delta) M+\delta^{T+1} V_{i}\left(\widehat{\pi}^{(i)}\right)\right)>0 .
\end{aligned}
$$

If $t \leq T$, then again using (28) and (30) we have

$$
\begin{aligned}
& V_{i}^{t}\left(\pi^{(j)}\right)-\left((1-\delta) \max _{s_{i}} u_{i}\left(s_{i}, m_{-i}^{j}\right)+\delta V_{i}\left(\pi^{(i)}\right)\right) \\
& =\left(1-\delta^{T+1-t}\right) u_{i}\left(m^{j}\right)+\delta^{T+1-t} V_{i}\left(\widehat{\pi}^{(j)}\right)-\left((1-\delta) \max _{s_{i}} u_{i}\left(s_{i}, m_{-i}^{j}\right)+\delta^{T+1} V_{i}\left(\widehat{\pi}^{(i)}\right)\right) \\
& >-\left(1-\delta^{T+1-t}\right) M+\delta^{T+1-t}\left(\alpha_{i}^{\prime}+\frac{\gamma}{4}\right)-\left((1-\delta) M+\delta^{T+1} \alpha_{i}^{\prime}\right) \\
& >-\left(1-\delta^{T}\right) M+\delta^{T}\left(\alpha_{i}^{\prime}+\frac{\gamma}{4}\right)-\left((1-\delta) M+\delta^{T+1} \alpha_{i}^{\prime}\right) \\
& >-\left(1-\delta^{T}\right) M+\delta^{T} \frac{\gamma}{4}-(1-\delta) M .
\end{aligned}
$$

(The last inequality in the above expression follows from $\alpha_{i}^{\prime}>0$.) But by (22) we have $\delta^{T} \frac{\gamma}{4}>$ $2\left(1-\delta^{T}\right) M>(1-\delta) M+\left(1-\delta^{T}\right) M$. Thus,

$$
\inf _{t}\left[V_{i}^{t}\left(\pi^{(j)}\right)-\left((1-\delta) \max _{s_{i}} u_{i}\left(s_{i}, \pi_{-i}^{(j), t}\right)+\delta V_{i}\left(\pi^{(i)}\right)\right)\right]>0 .
$$

This shows that that the simple strategy described by $\left(\pi^{(0)}, \pi^{(1)}, \ldots, \pi^{(n)}\right)$ above is strictly enforceable. Since $\pi^{(0)}=\pi$ this completes the proof of the Proposition for the case of $n \geq 3$.

Case B: $n=2$.

Let $\bar{m}=\left(m_{1}^{2}, m_{2}^{1}\right)$ be the mutual minmax profile. Clearly, $v_{i} \geq u_{i}(\bar{m})$. For convenience, in this case we normalize payoffs so that $u_{i}(\bar{m})=0$ for both $i=1,2$.

Let $\alpha \in \mathcal{U}^{0}$. Since $\mathcal{U}^{0}$ is convex and $\alpha_{i}>v_{i} \geq u_{i}(\bar{m})=0$ for all $i$, there exists $y \in \mathcal{U}^{0}$ such that $\alpha_{i}>y_{i}>v_{i}$. Fix any such $y$. 
Define $\varepsilon_{i}=\min \left\{\alpha_{i}-y_{i}, y_{i}-v_{i}\right\}>0$ and $\gamma=\min _{i} \varepsilon_{i} / 4$. Fix any $\xi>0$ such that

$$
\xi<\min \left\{\gamma, \frac{\gamma^{2}}{2 M}\right\}
$$

where, as before, $M=\max _{i=1,2} \max _{s}\left|u_{i}(s)\right|$. Clearly, the following two conditions hold:

$$
\begin{aligned}
& y_{i}-\xi>v_{i}+3 \gamma, \text { for all } i \\
& y_{i}+\xi<\alpha_{i}, \text { for all } i .
\end{aligned}
$$

Let $K \in \mathbb{N}$ be such that $D \subseteq \cup_{x \in D_{K}} B_{\xi / 2}(x)$ (see Sorin (1992, Proposition 1.3.)), $T \in \mathbb{N}$ be such that

$$
T>\frac{M}{\gamma}
$$

and $\bar{\delta} \in(0,1)$ be such that for all $\delta \in[\bar{\delta}, 1)$

$$
\begin{aligned}
& \frac{1-\delta^{T+1}}{1-\delta}>T, \\
& \delta^{T}>\frac{M}{M+\gamma}, \\
& 1-\delta<\frac{\gamma}{2 M} \text { and } \\
& \sup _{x \in[-M, M]^{K}}\left|\frac{1-\delta}{1-\delta^{K}} \sum_{k=1}^{K} \delta^{k-1} x_{k}-\frac{1}{K} \sum_{k=1}^{K} x_{k}\right|<\frac{\xi}{2} .
\end{aligned}
$$

Now fix any $\delta \geq \bar{\delta}$ and consider any $u \in C(\alpha, \delta)$. We will show that there is a $1-$ memory SPE strategy $f$ with $U(f, \delta)=u$.

Let $\pi \in \Lambda(\alpha, \delta)$ be such that it satisfies $V_{i}(\pi, \delta)=u_{i}$ for all $i \in N$. Since $\pi$ is a confusion-proof single path, by Proposition 2, to complete the proof and show that $u$ can be sustained by a 1 - memory SPE it is sufficient to establish that there exists a strictly enforceable simple strategy profile given by $\left(\pi^{(0)}, \pi^{(1)}, \pi^{(2)}\right)$ such that $\pi^{(0)}=\pi$ and $\pi^{(1)}=\pi^{(2)}$.

We first start by constructing a common punishment path $\bar{\pi}=\pi^{(1)}=\pi^{(2)}$. This path consists of playing $\bar{m}$ for the first $T$ periods followed by a path $\hat{\pi}$ yielding a payoff to each player $i$ that is less than $\alpha_{i}$ and bounded away from the minmax payoff $v_{i}$ by $\gamma$. Furthermore, $\hat{\pi}$ must be such that $V_{i}^{t}(\hat{\pi}) \geq V_{i}(\hat{\pi})$ for all $i$ and $t$ to prevent player $i$ to deviate at latter stages of his punishment path.

By (38), let $x \in D_{K}$ be such that $\|x-y\|<\xi / 2$. Let $\left\{s^{k}\right\}_{k=1}^{K}$ be such that

$$
\frac{1}{K} \sum_{k=1}^{K} u_{i}\left(s^{k}\right)=x_{i}
$$


for all $i \in N$. Then, let $\tilde{\pi}$ consist of repetitions of $\left(s^{1}, \ldots, s^{K}\right)$ and let

$$
z_{i}=V_{i}(\tilde{\pi})
$$

for all $i \in N$. Note that $\left\|x-V^{t}(\tilde{\pi})\right\|<\xi / 2$ for all $t \in \mathbb{N}$ by (38). In particular, we have that $\|x-z\|<\xi / 2$ and $\left\|z-V^{t}(\tilde{\pi})\right\| \leq\|z-x\|+\left\|x-V^{t}(\tilde{\pi})\right\|<\xi$ and so

$$
V_{i}^{t}(\tilde{\pi})>z_{i}-\xi \text {, for all } t \text { and } i
$$

Furthermore,

$$
\alpha_{i}>z_{i}>v_{i}+2 \gamma, \text { for all } i \text {. }
$$

The first inequality in (40) follows from $z_{i}=V_{i}(\tilde{\pi})<x_{i}+\frac{\xi}{2}<y_{i}+\xi$ and condition (33), and the second follows from $z_{i}>x_{i}-\xi>x_{i}-\gamma>y_{i}-\xi-\gamma$ and condition (32).

We now define $\hat{\pi}$ as follows: it consists of playing $\bar{m}$ for the first $R$ periods, for some some $R \in \mathbb{N}$ satisfying

$$
v_{i}+\gamma<\delta^{R} z_{i}<z_{i}-\xi, \text { for all } i=1,2,
$$

followed by playing the sequence $\left\{s^{1}, \ldots, s^{K}\right\}$ repeatedly. Thus,

$$
\hat{\pi}^{t}= \begin{cases}\bar{m} & \text { if } t \leq R \\ \tilde{\pi}^{t-T} & \text { if } t>R\end{cases}
$$

Before proceeding further with the construction of equilibrium strategy, we shall next establish the existence of a number $R \in \mathbb{N}$ with the above property in the following claim.

Claim 1 There exists $R \in \mathbb{N}$ satisfying (41).

Proof of Claim 1. Let

$$
a=\max _{i} \frac{v_{i}+\gamma}{z_{i}}, \quad b=1-\frac{\xi}{\min _{i} v_{i}+\gamma} \quad \text { and } l=b-a .
$$

Then, it follows that

$$
l=1-\max _{i} \frac{v_{i}+\gamma}{z_{i}}-\frac{\xi}{\min _{i} v_{i}+\gamma}>\frac{\gamma}{M}-\frac{\xi}{\min _{i} v_{i}+\gamma} \geq \frac{\gamma}{M}-\frac{\xi}{\gamma} \geq \frac{\gamma}{M}-\frac{\gamma}{2 M}=\frac{\gamma}{2 M}>0 .
$$

The first and the third inequality in the above follow respectively from $z_{i}>v_{i}+2 \gamma($ by (40)) and $\xi<\frac{\gamma^{2}}{2 M}$ (by the definition of $\xi$ ). This, together with $\frac{\gamma}{2 M}>1-\delta$ (condition (37)), imply that

$$
l>1-\delta \geq \delta^{r}(1-\delta)=\delta^{r}-\delta^{r+1} \text { for all } r \in \mathbb{N}_{0}
$$


Next, we show that there exists $R \in \mathbb{N}$ such that $\delta^{R} \in(a, b)$. First, note that $\delta^{0}=1>b$ and $\delta^{L}<a$ for $L$ sufficiently large. Let $r$ be the smallest integer in $\mathbb{N}_{0}$ such that $\delta^{r} \geq b$. Then $\delta^{r+1}<b$, by definition. Moreover, $\delta^{r+1}>a$; otherwise, $\delta^{r}-\delta^{r+1} \geq b-a=l$, but this contradicts (42). Thus, $\delta^{r+1} \in(a, b)$.

Now since $\delta^{R} \in(a, b)$, it then follows that for any $i=1,2$

$$
\frac{v_{i}+\gamma}{z_{i}} \leq \max _{j} \frac{v_{j}+\gamma}{z_{j}}<\delta^{R}<1-\frac{\xi}{\min _{j} v_{j}+\gamma} \leq 1-\frac{\xi}{v_{i}+\gamma}
$$

Hence, $v_{i}+\gamma<\delta^{R} z_{i}$. Also, since by $(40) z_{i}>v_{i}+2 \gamma$, it follows that $\delta^{R}<1-\frac{\xi}{z_{i}}$; so $\delta^{R} z_{i}<z_{i}-\xi$.

Next define the common punishment path $\bar{\pi}$ by

$$
\bar{\pi}^{t}= \begin{cases}\bar{m} & \text { if } t \leq T, \\ \hat{\pi}^{t-T} & \text { if } t>T\end{cases}
$$

Note that $V_{i}^{T+1}(\bar{\pi})=V_{i}(\hat{\pi})=\delta^{R} z_{i}$. Thus, we have from (41) and $\alpha_{i}>z_{i}$ (by (40)) that

$$
\alpha_{i}>V_{i}(\hat{\pi})>v_{i}+\gamma, \text { for any } i=1,2
$$

Also, by (39) and (41)

$$
V_{i}^{t}(\bar{\pi})=V_{i}^{t-T}(\widehat{\pi}) \geq V_{i}(\widehat{\pi}) \text { for any } t>T \text { and } i=1,2
$$

Now using the properties described in (43) and (44) we will next show that the simple strategy $\left(\pi^{(0)}, \pi^{(1)}, \pi^{(2)}\right)$ where $\pi^{(0)}=\pi$ and $\pi^{(i)}=\bar{\pi}$ for all $i=1,2$ supports $u$ as a strictly enforceable SPE.

Fix any $i=1,2$. First, consider player $i$ deviating from the equilibrium path $\pi^{(0)}=\pi$ in period

$t$. Then we have

$$
\begin{aligned}
& V_{i}^{t}(\pi)-\left((1-\delta) \max _{s_{i}} u_{i}\left(s_{i}, \pi_{-i}^{t}\right)+\delta V_{i}(\bar{\pi})\right) \\
& =V_{i}^{t}(\pi)-\left((1-\delta) \max _{s_{i}} u_{i}\left(s_{i}, \pi_{-i}^{t}\right)+\delta^{T+1} V_{i}(\widehat{\pi})\right) \\
& >\alpha_{i}-\left((1-\delta) M+\delta^{T+1} \alpha_{i}\right) .
\end{aligned}
$$

(The equality in the above follows from $V_{i}(\bar{\pi})=\delta^{T} V_{i}(\widehat{\pi})$, the inequality from $\pi \in \Lambda(\alpha, \delta)$ and (43).) Since $\alpha_{i}>(1-\delta) M+\delta^{T+1} \alpha_{i}$ is equivalent to $\frac{1-\delta^{T+1}}{1-\delta}>\frac{M}{\alpha_{i}}$, and

$$
\frac{1-\delta^{T+1}}{1-\delta}>T>\frac{M}{\gamma} \geq \frac{M}{\alpha_{i}}
$$


(which holds by (34), (35) and since $\alpha_{i}-v_{i} \geq \alpha_{i}-y_{i}>\gamma$ ), it follows that

$$
\inf _{t}\left[V_{i}^{t}(\pi)-\left((1-\delta) \max _{s_{i}} u_{i}\left(s_{i}, \pi_{-i}^{t}\right)+\delta V_{i}(\bar{\pi})\right)\right]>0 .
$$

Second, consider a deviation by $i$ from $\bar{\pi}^{t}$. If $t \leq T$ we have that

$$
\begin{aligned}
& V_{i}^{t}(\bar{\pi})-\left((1-\delta) \max _{s_{i}} u_{i}\left(s_{i}, m_{-i}^{i}\right)+\delta V_{i}(\bar{\pi})\right) \\
& =\delta^{T+1-t} V_{i}(\widehat{\pi})-(1-\delta) v_{i}+\delta^{T+1} V_{i}(\widehat{\pi}) \\
& \geq \delta^{T} V_{i}(\widehat{\pi})-\left((1-\delta) v_{i}+\delta^{T+1} V_{i}(\widehat{\pi})\right) .
\end{aligned}
$$

Since, by (36) and (43), $\delta^{T}>\frac{M}{M+\gamma} \geq \frac{v_{i}}{v_{i}+\gamma}>\frac{v_{i}}{V_{i}(\widehat{\pi})}$, it follows that $\delta^{T} V_{i}(\widehat{\pi})-\left((1-\delta) v_{i}+\delta^{T+1} V_{i}(\widehat{\pi})\right)>$ 0 .

If $t>T$ we have from (44) that

$$
V_{i}^{t}(\bar{\pi})-\left((1-\delta) \max _{s_{i}} u_{i}\left(s_{i}, \bar{\pi}_{-i}^{t}\right)+\delta V_{i}(\bar{\pi})\right) \geq V_{i}(\widehat{\pi})-\left((1-\delta) M+\delta^{T+1} V_{i}(\widehat{\pi})\right)
$$

Since $V_{i}(\widehat{\pi})>(1-\delta) M+\delta^{T+1} V_{i}(\widehat{\pi})$ is equivalent to $\frac{1-\delta^{T+1}}{1-\delta}>\frac{M}{V_{i}(\widehat{\pi})}$, and

$$
\frac{1-\delta^{T+1}}{1-\delta}>T>\frac{M}{\gamma}>\frac{M}{V_{i}(\widehat{\pi})}
$$

(which holds which holds by (34), (35) and (43)), it follows that

$$
\inf _{t}\left[V_{i}^{t}(\bar{\pi})-\left((1-\delta) \max _{s_{i}} u_{i}\left(s_{i}, \bar{\pi}_{-i}^{t}\right)+\delta V_{i}(\bar{\pi})\right)\right]>0 .
$$

This concludes the proof that the simple strategy described above by $\left(\pi^{(0)}, \pi^{(1)}, \pi^{(2)}\right)$ with $\pi^{(0)}=\pi$ and $\pi^{(1)}=\pi^{(2)}=\bar{\pi}$ is strictly enforceable and induces a payoff of $u$. This completes the proof of the case $n=2$ and, thus, of Proposition 3.

Proof of Lemma 2. Fix any $u \in \mathcal{U}$ and any $\zeta>0$. Now we make two claims.

Claim 2 There exists $y, \alpha \in \mathcal{U}^{0}$ such that $\|y-u\|<\zeta / 4$ and $y_{i}>\alpha_{i}$ for all $i$.

We shall prove this Claim 2 for the two cases of $\operatorname{dim}(\mathcal{U})=n$ and $n=2$ separately.

Case A: $\operatorname{dim}(\mathcal{U})=n$.

In this case we shall first prove that there exists $\bar{u} \in \operatorname{int}\left(\mathcal{U}^{0}\right)$. By Theorem 6.2 in Rockafellar (1970), $\operatorname{dim}(\mathcal{U})=n$ implies that there exists $u \in \operatorname{int}(\mathcal{U})$. Let $\varepsilon>0$ be such that $B_{\varepsilon}(u) \subseteq \mathcal{U}$. Define 
$\bar{u}$ by $\bar{u}_{i}=u_{i}+\varepsilon / 2$ for all $i$. Note that $B_{\varepsilon / 2}(\bar{u}) \subseteq \operatorname{int}\left(\mathcal{U}^{0}\right)$ : if $\|\tilde{u}-\bar{u}\|<\varepsilon / 2$, then $\|\tilde{u}-u\|<\varepsilon$ and so $\tilde{u} \in \mathcal{U}$; furthermore, $\tilde{u}_{i}>\bar{u}_{i}-\varepsilon / 2=u_{i} \geq v_{i}$ and so $\tilde{u} \in \mathcal{U}^{0}$. This implies that $\bar{u} \in \operatorname{int}\left(\mathcal{U}^{0}\right)$.

Next, we show that there exists $y \in \operatorname{int}\left(\mathcal{U}^{0}\right)$ such that $\|y-u\|<\zeta / 4$. Define $u^{k}=\frac{1}{k} \bar{u}+\left(1-\frac{1}{k}\right) u$. Since $u_{i}^{k}>v_{i}$ for all $i, u^{k} \in \mathcal{U}^{0}$. Furthermore, by Theorem 6.1 in Rockafellar $(1970) u^{k} \in \operatorname{int}(\mathcal{U})$. So $B_{\rho}\left(u^{k}\right) \subseteq \mathcal{U}^{0}$ for some $\rho>0$, i.e., $u^{k} \in \operatorname{int}\left(\mathcal{U}^{0}\right)$. Since $u^{k} \rightarrow u$, there exists $k^{\prime}$ such that $\left\|u^{k^{\prime}}-u\right\|<\zeta / 4$. Denote $u^{k^{\prime}}$ by $y$. Then $y \in \operatorname{int}\left(\mathcal{U}^{0}\right)$ and $\|y-u\|<\zeta / 4$.

Finally, the existence of $\alpha \in \mathcal{U}^{0}$ such that $y_{i}>\alpha_{i}$ for all $i$ follows immediately from $y \in \operatorname{int}\left(\mathcal{U}^{0}\right)$.

Case B: $n=2$ and $\mathcal{U}^{0} \neq \emptyset$.

Then there exists $\bar{u} \in \mathcal{U}^{0}$ such that $\bar{u}_{i}>v_{i}$, for all $i$. This, together with $u_{i} \geq v_{i}$, imply that there exists $y \in \mathcal{U}^{0}$ such that $\|y-u\|<\zeta / 4$ (take an appropriate convex combination of $\bar{u}$ and $u)$. Also, since $y_{i}>v_{i} \geq u_{i}(\bar{m})$ for all $i$, there exists $\lambda \in(0,1)$ such that $\lambda y+(1-\lambda) u(\bar{m}) \in \mathcal{U}^{0}$. Denote $\lambda y+(1-\lambda) u(\bar{m})$ by $\alpha$. Then, $\alpha \in \mathcal{U}^{0}$ and $y_{i}>\alpha_{i}$ for all $i$.

Claim 3 For any $y \in \mathcal{U}^{0}$ and $\xi>0$, there exists $\tilde{\delta} \in(0,1)$ and a confusion proof single path $\tilde{\pi}$ such that $\left\|V^{t}(\tilde{\pi})-y\right\|<3 \xi$ for all $t \in N$ and for all $\delta \geq \tilde{\delta}$.

In order to prove Claim 3, fix any $y \in \mathcal{U}^{0}$ and $\xi>0$. Let $K \in \mathbb{N}$ be such that $D \subseteq \cup_{x \in D_{K}} B_{\xi}(x)$ and $\tilde{\delta} \in(0,1)$ be such that for all $\delta \geq \tilde{\delta}$

$$
\sup _{x \in[-M, M]^{K}}\left|\frac{1-\delta}{1-\delta^{K}} \sum_{k=1}^{K} \delta^{k-1} x_{k}-\frac{1}{K} \sum_{k=1}^{K} x_{k}\right|<\xi .
$$

Let $\delta \geq \tilde{\delta}$. Let $\left\{\bar{s}^{k}\right\}_{k=1}^{K}$ be such that

$$
\left\|\frac{1}{K} \sum_{k=1}^{K} u\left(\bar{s}^{k}\right)-y\right\|<\xi .
$$

Since $S_{i}$ is connected for all $i$, there exist a (finite) sequence $\left\{s^{k}\right\}_{k=1}^{K}$ such that $\left|u_{i}\left(s^{t}\right)-u_{i}\left(\bar{s}^{t}\right)\right|<\xi$ for all $i$ and $t$ and $s_{i}^{t} \neq s_{j}^{r}$ for all $1 \leq t, r \leq K$ and $1 \leq i, j \leq n$ satisfying $(i, t) \neq(j, r)$. Then,

$$
\left\|\frac{1}{K} \sum_{k=1}^{K} u\left(s^{k}\right)-\frac{1}{K} \sum_{k=1}^{K} u\left(\bar{s}^{k}\right)\right\|<\xi
$$

and so

$$
\left\|\frac{1}{K} \sum_{k=1}^{K} u\left(s^{k}\right)-y\right\|<2 \xi
$$


Let $\tilde{\pi}$ be the repetition of $\left\{s^{k}\right\}$. Then $\left\|V^{t}(\tilde{\pi})-y\right\|<3 \xi$ for all $t \in \mathbb{N}$. This completes the proof of Claim 3.

Now to complete the proof of Lemma 2, note that it follows from Claim 2 above that there exists $y$ and $\alpha \in \mathcal{U}^{0}$ such that $\|y-u\|<\zeta / 4$ and $y_{i}>\alpha_{i}$ for all $i$. Next, consider any $\xi>0$ such that $\xi<\frac{\zeta}{4}$ and $y_{i}-3 \xi>\alpha_{i}$ for all $i$. Then by Claim 3 above there exist $\tilde{\delta} \in(0,1)$ and a confusion proof single path $\tilde{\pi}$ such that $\left\|V^{t}(\tilde{\pi})-y\right\|<3 \xi$ for all $t \in \mathbb{N}$ and for all $\delta>\tilde{\delta}$. Now fix any $\delta>\tilde{\delta}$ and let $\tilde{u}=V(\tilde{\pi})$. Then $\|\tilde{u}-u\| \leq\|u-y\|+\|\tilde{u}-y\|<\zeta / 4+3 \zeta / 4=\zeta$ and $V_{i}^{t}(\tilde{\pi})>y_{i}-3 \xi>\alpha_{i}$ for all $i$ and $t \in \mathbb{N}$. But then $\tilde{u} \in C(\alpha, \delta)$.

This completes the proof of Lemma 2.

Proof of Proposition 4. Let $u \in \mathcal{U}^{0} \cap C$. Then there exists $\varepsilon>0$ be such that

$$
u_{i}>v_{i}+\varepsilon \text { for all } i \in N
$$

Moreover, since $u \in \mathcal{U}^{0} \cap C$ there exists a confusion proof path $\pi^{(0)}$ that consists of repeatedly playing a finite sequence of action profiles $\left\{\bar{s}^{k}\right\}_{k=1}^{K}$ such that $u=\frac{1}{K} \sum_{k=1}^{K} u\left(\bar{s}^{k}\right)$ for all $i$.

Let $\nu_{i}: S \rightarrow \mathbb{R}$ be defined by

$$
\nu_{i}(s)=\max _{\tilde{s}_{i} \in S_{i}} u_{i}\left(\tilde{s}_{i}, s_{-i}\right)
$$

Also, define $M_{i}=\max _{s \in S}\left|u_{i}(s)\right|$. Then, let $R \in \mathbb{N}$ be such that

$$
\frac{R \varepsilon}{2}>\left(M_{i}-u_{i}\right) K, \text { for all } i \in N
$$

Since $S$ is connected and $\nu_{i}$ is continuous (by the continuity of $u_{i}$ and compactness of $S_{i}$ ), then $\nu_{i}(S) \subseteq \mathbb{R}$ is also connected. Connectedness of $S$ and $\nu_{i}(S)$, together with (46) and $v_{i}=\nu_{i}\left(m^{i}\right)$, imply that for each $i \in N$ there exists a set $\left\{b^{(i), t}\right\}_{t=1}^{R} \subseteq S$ such that

$$
\begin{aligned}
& b_{l}^{(i), r} \neq \pi_{l}^{(0), t} \text { for all } i, l \in N, t \in \mathbb{N} \text { and } 1 \leq r \leq R, \\
& b_{l}^{(i), r} \neq b_{l}^{(j), q} \text { for all } i, j, l \in N, \text { and } 1 \leq r, q \leq R \text { such that }(i, r) \neq(j, q) \\
& u_{i} \geq \nu_{i}\left(b^{(i), r}\right)+\frac{\varepsilon}{2} \text { for all } 1 \leq r \leq R \\
& \text { if } n=2 \text { then } b^{(i)}=b^{(j)}
\end{aligned}
$$

By the connectedness of $S$ and continuity of $v_{i}$, the above four conditions hold because for $n>2$ there exists a continnum of actions profiles $B^{i} \subseteq S$ such that $\left\|\nu_{i}(b)-v_{i}\right\|<\varepsilon / 2$ for all $i$ and $b \in B^{i}$, 
and when $n=2$ there exists a continnum of actions profiles $B$ such that $\left\|\nu_{i}(b)-\pi_{i}\left(m_{1}^{2}, m_{2}^{1}\right)\right\|<\varepsilon / 2$, for all $i=1,2$, where as before $\left(m_{1}^{2}, m_{2}^{1}\right)$ is the mutual minmax strategies.

We next show that

$$
\frac{1}{q+R}\left[\nu_{i}\left(\pi^{(0), q}\right)+\sum_{r=1}^{q-1} u_{i}\left(\pi^{(0), r}\right)+\sum_{r=1}^{R} u_{i}\left(b^{(i), r}\right)\right] \leq u_{i}
$$

for all $1 \leq i \leq n$ and $q \in \mathbb{N}$. To show this let $q=m+d$ where $m, d \in \mathbb{N}_{0}, m$ is a multiple of $K$ and $0 \leq d<K$. Then, by condition (47) and (50), we have

$$
\begin{aligned}
& \frac{1}{q+R}\left[\nu_{i}\left(\pi^{(0), q}\right)+\sum_{r=1}^{q-1} u_{i}\left(\pi^{(0), r}\right)+\sum_{r=1}^{R} u_{i}\left(b^{(i), r}\right)\right]= \\
& \frac{1}{m+d+R}\left[\nu_{i}\left(\pi^{(0), q}\right)+m u_{i}+\sum_{r=1}^{d-1} u_{i}\left(\pi^{(0), r}\right)+\sum_{r=1}^{R} u_{i}\left(b^{(i), r}\right)\right] \leq \\
& \frac{1}{m+d+R}\left[d M_{i}+m u_{i}+\sum_{r=1}^{R} u_{i}\left(b^{(i), r}\right)\right]< \\
& \frac{1}{m+d+R}\left[d u_{i}+\frac{R \varepsilon}{2}+m u_{i}+R\left(u_{i}-\frac{\varepsilon}{2}\right)\right] \leq u_{i} .
\end{aligned}
$$

Furthermore, since $u_{i}(a) \leq \nu_{i}(a)$ for any $a$, it follows from (50) that

$$
\frac{1}{t}\left[\nu_{i}\left(b^{(i), t}\right)+\sum_{r=1}^{t-1} u_{i}\left(b^{(i), r}\right)\right]+\frac{\varepsilon}{2} \leq u_{i} \text {, for all } 1 \leq i \leq n \text { and } 1 \leq t \leq R .
$$

Next, for all $1 \leq i \leq n$, define the path $\pi^{(i)}$ as follows:

$$
\pi^{(i), t}= \begin{cases}b^{(i), t} & \text { if } t \leq R \\ \pi^{(0), t-R} & \text { if } t>R\end{cases}
$$

Let $f$ be the strategy profile defined by $\left(\pi^{(0)}, \ldots, \pi^{(n)}\right)$. Since $\pi^{(0)}$ is confusion proof single path it follows from Proposition 1, (48) and (49) that $f$ has 1 - memory. Using (52) and (53), we now show that $f$ is a SPE.

Consider any history $h=\left(s^{1}, \ldots, s^{t-1}\right)$. Since $f \mid h$ induces the same outcome path as $\pi^{(0)}$ at some stage it follows that $U_{i}^{\infty}(f \mid h)=u_{i}$, for all $i$.

Next consider any deviation by player $i$ to another strategy $f_{i}^{\prime}$ at $h$. Let $\pi\left(f_{i}^{\prime}, f_{-i} \mid h\right)=\left\{\tilde{s}^{\tau}\right\}_{\tau=t}^{\infty}$. Since players use the limit of the mean criterion, it is enough to consider the case in which $f_{i}^{\prime}$ deviates from $f_{i}$ infinitely often in the subgame defined by $h$. Thus, suppose that there is an infinite sequence $\left\{\mu^{\tau}\right\}_{\tau \in \mathbb{N}}$ with $\mu^{\tau} \geq t$, such that: 
1. either $\left[\tilde{s}_{-i}^{\mu^{\tau}}=\pi_{-i}^{(0), k}\right.$ and $\tilde{s}_{i}^{\mu^{\tau}} \neq \pi_{i}^{(0), k}$ for some $\left.k \in \mathbb{N}\right]$ or $\left[\tilde{s}_{-i}^{\mu^{\tau}}=b_{-i}^{(i), r}\right.$ and $\left.\tilde{s}_{i}^{\mu^{\tau}} \neq b_{i}^{(i), r}\right]{ }^{15}$

2. $\tilde{s}^{\mu^{\tau}+r}=b^{(i), r}$ if $1 \leq r<\min \left\{\mu^{\tau+1}-\mu^{\tau}, R+1\right\}$.

3. $\tilde{s}^{\mu^{\tau}+r}=\pi^{(0), r-R}$ if $R+1 \leq r<\mu^{\tau+1}-\mu^{\tau}$.

Then,

$$
\begin{aligned}
& U_{i}^{\infty}\left(f_{i}^{\prime}, f_{-i} \mid h\right)=\liminf _{n \rightarrow \infty} \frac{1}{n+1} \sum_{\mu=0}^{n} u_{i}\left(\tilde{s}^{t+\mu}\right) \\
& =\liminf _{n \rightarrow \infty} \frac{1}{\mu^{n+1}-t+1}\left(\sum_{\mu=t}^{\mu^{1}} u_{i}\left(\tilde{s}^{\mu}\right)+\sum_{\tau=1}^{n} \sum_{\mu=\mu^{\tau}+1}^{\mu^{\tau+1}} u_{i}\left(\tilde{s}^{\mu}\right)\right)=\liminf _{n \rightarrow \infty} \frac{1}{\mu^{n+1}-\mu^{1}} \sum_{\tau=1}^{n} \sum_{\mu=\mu^{\tau}+1}^{\mu^{\tau+1}} u_{i}\left(\tilde{s}^{\mu}\right) .
\end{aligned}
$$

Now for any $\tau$, if $\mu^{\tau+1}-\mu^{\tau}=r \leq R$, then

$$
\sum_{\mu=\mu^{\tau}+1}^{\mu^{\tau+1}} u_{i}\left(\tilde{s}^{\mu}\right) \leq \sum_{k=1}^{r-1}\left(u_{i}\left(b^{(i), k}\right)+\nu_{i}\left(b^{(i), r}\right)\right) \leq r u_{i}
$$

where the second inequality follows from (53). If $\mu^{\tau+1}-\mu^{\tau}>R$, then

$$
\sum_{\mu=\mu^{\tau}+1}^{\mu^{\tau+1}} u_{i}\left(\tilde{s}^{\mu}\right) \leq \sum_{r=1}^{R} u_{i}\left(b^{(i), r}\right)+\sum_{r=1}^{\mu^{\tau+1}-\mu^{\tau}-R-1} u_{i}\left(\pi^{(0), r}\right)+\nu_{i}\left(\pi^{(0), \mu^{\tau+1}-\mu^{\tau}-R}\right) \leq\left(\mu^{\tau+1}-\mu^{\tau}\right) u_{i},
$$

where the last inequality follows from (52). Therefore, $\left.U_{i}^{\infty}\left(\left(f_{i}^{\prime}, f_{-i} \mid h\right)\right) \leq u_{i} \leq U_{i}^{\infty}(f \mid h)\right)$. This completes the proof of Theorem 4

Proof of Theorem 3. Let $u \in \mathcal{U}$ and $\zeta>0$. Since $\mathcal{U}^{0}$ is nonempty, there exists $y \in \mathcal{U}^{0}$ and $\xi>0$ such that $\|u-y\|<\xi, y_{i}-v_{i}>\xi$ for all $i$ and $\xi \leq \zeta / 2$ (let $x \in \mathcal{U}^{0}$ and consider $y=\lambda u+(1-\lambda) x$ for some $\lambda \in(0,1)$ sufficiently close to 1$)$.

Let $K \in \mathbb{N}$ be such that $D \subseteq \cup_{x \in D_{K}} B_{\xi / 2}(x)$. Then there exists a sequence $\left\{\bar{s}^{k}\right\}_{k=1}^{K}$ be such that

$$
\left\|\frac{1}{K} \sum_{k=1}^{K} u\left(\bar{s}^{k}\right)-y\right\|<\frac{\xi}{2} .
$$

Since $S_{i}$ is connected for all $i$, there exist a (finite) sequence $\left\{s^{k}\right\}_{k=1}^{K}$ such that $\left|u_{i}\left(s^{k}\right)-u_{i}\left(\bar{s}^{k}\right)\right|<\xi / 2$ for all $i$ and $k$ and $s_{i}^{t} \neq s_{j}^{r}$ for all $1 \leq t, r \leq K$ and $1 \leq i, j \leq n$ satisfying $(i, t) \neq(j, r)$. Then,

$$
\left\|\frac{1}{K} \sum_{k=1}^{K} u\left(s^{k}\right)-\frac{1}{K} \sum_{k=1}^{K} u\left(\bar{s}^{k}\right)\right\|<\frac{\xi}{2}
$$

${ }^{15}$ If $\tau=1$ it can also be that $\tilde{s}_{-i}^{\mu^{1}}=\pi_{-i}^{(j), k}$ and $\tilde{s}_{i}^{\mu^{1}} \neq \pi_{i}^{(j), k}$ for some $k \in \mathbb{N}$ and $j \notin\{0, i\}$. 
and so

$$
\left\|\frac{1}{K} \sum_{k=1}^{K} u\left(s^{k}\right)-y\right\|<\xi .
$$

Finally, let $\tilde{\pi}$ be the repetition of $\left\{s^{k}\right\}_{k=1}^{K}$ and $\tilde{u}=V^{\infty}(\tilde{\pi})=\sum_{k=1}^{K} u\left(s^{k}\right) / K$. Then, $\|u-\widetilde{u}\|<2 \xi$ and $\widetilde{u}_{i}>v_{i}$; hence $\tilde{u} \in \mathcal{U}^{0} \cap C$. Thus, by Theorem 4 , there exists a 1 - memory SPE strategy profile $f$ with $U^{\infty}(f)=\tilde{u}$. Moreover, since $\|\tilde{u}-u\|<2 \xi \leq \zeta$, it follows that $\left\|U^{\infty}(f)-u\right\|<\zeta$.

Proof of Theorem 5. Let $u \in \operatorname{int}\left(\mathcal{U}^{0}\right)$ and $\alpha \in \operatorname{int}\left(\mathcal{U}^{0}\right)$ be such that $u_{i}>\alpha_{i}$ for all $i$. Since $\operatorname{int}\left(\mathcal{U}^{0}\right)$ is non-empty it follows that $\operatorname{dim}(\mathcal{U})=n$. Then, by Proposition 5 , there exists $\hat{\delta}$ such that that for all $\delta \geq \hat{\delta}$ the following holds: for all payoffs $u^{\prime} \in \tilde{C}(\alpha, \delta)$ there exists time dependent 1 - memory $\operatorname{SPE} f$ with $U(f)=u^{\prime}$. Let $\varepsilon=\min _{i}\left(u_{i}-v_{i}\right)$. Also, denote the discount factor corresponding to $\varepsilon$, given in Lemma 2 of Fudenberg and Maskin (1991), by $\bar{\delta}$.

Let $\delta^{*}=\max \{\hat{\delta}, \bar{\delta}\}$. Fix any $\delta>\delta^{*}$. Then, by the definition of $\bar{\delta}$ there exists a sequence of actions $\left\{s_{t}\right\}_{t=1}^{\infty}$ whose payoff is $u$ and whose continuation payoffs at each time are within $\varepsilon$ of $u$. This implies that the continuation payoffs of $\left\{s_{t}\right\}_{t=1}^{\infty}$ at each date are above $\alpha$. Thus, $u \in \tilde{C}(\alpha, \delta)$. Hence, since $\delta \geq \hat{\delta}$, by the definition of $\hat{\delta}$ there exists a time dependent 1 - memory SPE strategy $f$ such that $U(f)=u$.

\section{References}

Abreu, D. (1988): "On the Theory of Infinitely Repeated Games with Discounting," Econometrica, 56, 383-396.

Abreu, D., P. Dutta, and L. Smith (1994): "The Folk Theorem for Repeated Games: A Neu Condition," Econometrica, 62, 939-948.

Aumann, R. (1964): "Mixed and Behavioural Strategies in Infinite Extensive Games," in Advances in Game Theory, ed. by M. Dresher, L. Shapley, and A. Tucker. Princeton University Press, Princeton.

Aumann, R., And L. Shapley (1994): "Long-Term Competition - A Game-Theoretic Analysis," in Essays in Game Theory in Honor of Michael Maschler, ed. by N. Megiddo. Springer-Verlag, New York. 
Aumann, R., And S. Sorin (1989): "Cooperation and Bounded Recall," Games and Economic Behavior, 1, 5-39.

Barlo, M. (2003): "Essays in Game Theory," Ph.D. thesis, University of Minnesota.

Barlo, M., and G. Carmona (2006): "Time Dependent Bounded Recall Strategies Are Enough to Play the Discounted Repeated Prisoners' Dilemma," Sabancı University and Universidade Nova de Lisboa.

Bhaskar, V., and F. Vega-Redondo (2002): "Asynchronous Choice and Markov Equilibria," Journal of Economic Theory, 103, 334-350.

Fudenberg, D., And E. Maskin (1986): "The Folk Theorem in Repeated Games with Discounting or with Incomplete Information," Econometrica, 54, 533-554.

(1991): "On the Dispensability of Public Randomization in Discounted Repeated Games," Journal of Economic Theory, 53, 428-438.

Kalai, E., And W. Stanford (1988): "Finite Rationality and Interpersonal Complexity in Repeated Games," Econometrica, 56, 397-410.

LeHrer, E. (1988): "Repeated Games with Stationary Bounded Recall Strategies," Journal of Economic Theory, 46, 130-144.

(1994): "Finitely Many Players with Bounded Recall in Infinitely Repeated Games," Games and Economic Behavior, 6, 97-113.

Mailath, G., A. Postlewaite, and L. Samuelson (2005): “Contemporaneous Perfect EpsilonEquilibrium," Games and Economic Behavior, 53, 126-140.

Neyman, A., And D. Okada (1999): "Strategic Entropy and Complexity in Repeated Games," Games and Economic Behavior, 29, 191-223.

Rockafellar, R. T. (1970): Convex Analysis. Princeton University Press, Princeton.

Sabourian, H. (1989): "The Folk Theorem of Repeated Games with Bounded Recall (One-Period) Memory," Economic Theory Discussion Paper 143, University of Cambridge. 
(1998): "Repeated Games with $M$-period Bounded Memory (Pure Strategies)," Journal of Mathematical Economics, 30, 1-35.

SoRIn, S. (1992): "Bounded Rationality and Strategic Complexity in Repeated Games," in Handbook of Game Theory, Volume 1, ed. by R. Aumann, and S. Hart. Elsevier Science Publishers. 